DOI (Digital Object Identifier) 10.1007/s102310100025

Leonardo Colzani · Alice Cominardi · Krzysztof Stempak ${ }^{\star}$

\title{
Radial solutions to the wave equation
}

Received: July 7, 1998

Published online: March 19, 2002 - (C) Springer-Verlag 2002

Abstract. We study some boundedness properties of radial solutions to the Cauchy problem associated to the wave equation $\left(\partial_{t}^{2}-\triangle_{x}\right) u(t, x)=0$ and meanwhile we give a new proof of the solution formula.

Sunto. Studiamo delle proprietà di limitatezza per soluzioni radiali del problema di Cauchy associato all'equazione delle onde $\left(\partial_{t}^{2}-\triangle_{x}\right) u(t, x)=0$ e nel frattempo diamo anche una nuova dimostrazione della formula risolutiva per tale equazione.

Mathematics Subject Classification (2000). Primary: 35L05, 35Q05

Key words. wave equation - radial solutions - weak-type estimates

In this paper we want to prove some norm estimates for radial solutions to the wave equation and, in order to do this, we start by recalling the explicit form of these solutions.

Let us consider the Cauchy problem for the wave equation in $(t, x) \in R \times R^{D}$,

$$
(*)\left\{\begin{array}{l}
\frac{\partial^{2}}{\partial t^{2}} u(t, x)=\sum_{j=1}^{D} \frac{\partial^{2}}{\partial x_{j}^{2}} u(t, x), \\
u(0, x)=\Phi(x), \frac{\partial}{\partial t} u(0, x)=\Psi(x) .
\end{array}\right.
$$

The d'Alembert formula,

$$
u(t, x)=\frac{\Phi(x-t)+\Phi(x+t)}{2}+\frac{1}{2} \int_{x-t}^{x+t} \Psi(s) d s,
$$

gives the solution when the space dimension is one, while the Poisson formula,

$$
u(t, x)=\frac{\partial}{\partial t}\left\{\frac{t}{4 \pi} \int_{\{|y|=1\}} \Phi(x-t y) d \sigma(y)\right\}+\left\{\frac{t}{4 \pi} \int_{\{|y|=1\}} \Psi(x-t y) d \sigma(y)\right\},
$$

L. Colzani, A. Cominardi: Dipartimento di Matematica, Università degli Studi di MilanoBicocca, via Bicocca degli Arcimboldi 8, 20126 Milano, Italia,

e-mail: leonardo@matapp. unimib.it

K. Stempak: Instytut Matematyki Politechnika Wrocławska, Wyb. Wyspia'nskiego 27, 50-370 Wrocław, Poland, e-mail: stempak@im.pwr.wroc.pl

* The research of the third author was supported in part by the Polish grant KBN $n^{\circ} 2$ PO3A03009 and by the Italian CNR. 
gives the solution in dimension three. The form of solutions in an arbitrary dimension is due to Tedone (see [10]). When the dimension is odd, $D=2 N+1$, then

$$
\begin{aligned}
u(t, x)= & b(N) \frac{\partial}{\partial t}\left(\frac{1}{t} \frac{\partial}{\partial t}\right)^{N-1}\left\{t^{2 N-1} \int_{\{|y|=1\}} \Phi(x-t y) d \sigma(y)\right\} \\
& +b(N)\left(\frac{1}{t} \frac{\partial}{\partial t}\right)^{N-1}\left\{t^{2 N-1} \int_{\{|y|=1\}} \Psi(x-t y) d \sigma(y)\right\},
\end{aligned}
$$

where $b(N)=2^{-1}(1 \cdot 3 \cdot 5 \cdot \ldots \cdot(2 N-1))^{-1} \pi^{-N-1 / 2} \Gamma(N+1 / 2)$ and $d \sigma(y)$ is the surface measure on $\{|y|=1\}$. When the dimension is even, $D=2 N$, by the method of descent one has

$$
\begin{aligned}
u(t, x)= & 2 b(N) \frac{\partial}{\partial t}\left(\frac{1}{t} \frac{\partial}{\partial t}\right)^{N-1}\left\{t^{2 N-1} \int_{\{|y|<1\}} \frac{\Phi(x-t y)}{\sqrt{1-|y|^{2}}} d y\right\} \\
& +2 b(N)\left(\frac{1}{t} \frac{\partial}{\partial t}\right)^{N-1}\left\{t^{2 N-1} \int_{\{|y|<1\}} \frac{\Psi(x-t y)}{\sqrt{1-|y|^{2}}} d y\right\} .
\end{aligned}
$$

These formulas require approximately $D / 2$ derivatives of the initial conditions, hence they are not immediately applicable to non-smooth data. However, it is known that when these data are radial then there exists a solution formula with no derivatives involved. When the initial data are radial, writing $|x|=r, \Phi(x)=\phi(r)$, $\Psi(x)=\psi(r)$, with $\phi(r)$ and $\psi(r)$ even functions on $R$, and expressing the Laplacian in polar coordinates, one is led to the Cauchy problem

$$
(* *)\left\{\begin{array}{l}
\frac{\partial^{2}}{\partial t^{2}} u(t, r)=\frac{\partial^{2}}{\partial r^{2}} u(t, r)+\frac{D-1}{r} \frac{\partial}{\partial r} u(t, r), \\
u(0, r)=\phi(r), \frac{\partial}{\partial t} u(0, r)=\psi(r) .
\end{array}\right.
$$

Assume now $D=3$. Then one easily checks that $r \cdot u(t, r)$ satisfies the onedimensional wave equation $\frac{\partial^{2}}{\partial t^{2}}(r u(t, r))=\frac{\partial^{2}}{\partial r^{2}}(r u(t, r))$ with $r u(0, r)=r \phi(r)$ and $\frac{\partial}{\partial t}(r u(0, r))=r \psi(r)$. Hence, by d'Alembert's formula,

$$
u(t, r)=\frac{(r-t) \phi(r-t)+(r+t) \phi(r+t)}{2 r}+\frac{1}{2 r} \int_{r-t}^{r+t} s \psi(s) d s .
$$

Observe that since $s \psi(s)$ is an odd function, the above integration can be reduced to the interval $|r-t|<s<r+t$. This formula does not contain derivatives of the initial data, at least if $r \neq 0$, but taking the limit as $r \rightarrow 0+$ a derivative appears, $u(t, 0)=t \frac{\partial}{\partial t} \phi(t)+\phi(t)+t \psi(t)$.

For an arbitrary dimension the formula of solutions is more complicated. It involves terms of the form $r^{(1-D) / 2}|r \pm t|^{(D-1) / 2} \phi(r \pm t)$ and integrals of the 
initial data against hypergeometric functions, but when the space dimension is odd the hypergeometric functions reduce to polynomials and this makes things much simpler. In particular, when $D=2 N+1$, then the radial solutions to the wave equation $(* *)$ are given by

$$
\begin{aligned}
u(t, r)= & \frac{(r-t)^{N} \phi(r-t)+(r+t)^{N} \phi(r+t)}{2 r^{N}} \\
& -\frac{1}{2} t r^{-N-1} \int_{|r-t|}^{r+t} s^{N-1} \dot{\mathbb{P}}_{N-1}\left(\frac{s^{2}+r^{2}-t^{2}}{2 r s}\right) \phi(s) d s \\
& +\frac{1}{2} r^{-N} \int_{|r-t|}^{r+t} s^{N} \mathbb{P}_{N-1}\left(\frac{s^{2}+r^{2}-t^{2}}{2 r s}\right) \psi(s) d s
\end{aligned}
$$

where $\mathbb{P}_{N-1}(z)$ is the Legendre polynomial of degree $N-1$ and $\dot{\mathbb{P}}_{N-1}(z)$ is its derivative. Since $\mathbb{P}_{-1}(z)=\mathbb{P}_{0}(z)=1$, these formulas are consistent with d'Alembert's formulas of radial solutions to the wave equation in dimensions one and three.

As we have said, a solution formula for $(* *)$ was already known. Indeed, introducing the characteristic variables $\xi=r+t, \eta=r-t$, the wave equation reduces to the Euler-Poisson-Darboux equation,

$$
\frac{\partial^{2}}{\partial \xi \partial \eta} u(\xi, \eta)+\frac{(D-1) / 2}{\xi+\eta} \frac{\partial}{\partial \xi} u(\xi, \eta)+\frac{(D-1) / 2}{\xi+\eta} \frac{\partial}{\partial \eta} u(\xi, \eta)=0
$$

and a solution can be found by Riemann's method. See, for example, [3, V. 5] or [11]. Anyhow, we shall present a different derivation which is based on Fourier analysis, also because this will give us the opportunity to get familiar with some special functions and to study the singularities of the kernels that give the solutions. Indeed the main issue of the paper is on some norm estimates for radial solutions to the wave equation, and we shall accomplish this task via the explicit formula of solutions.

Since $|x|^{(1-D) / 2}|| x| \pm t|^{(D-1) / 2} \phi(|x| \pm t)$ are in some sense the main terms of radial solutions to $(*)$, it is easily seen that for a fixed time $t$ the inequality

$$
\left\{\int_{R^{D}}|u(t, x)|^{p} d x\right\}^{1 / p} \leq c\left\{\int_{R^{D}}|u(0, x)|^{p} d x\right\}^{1 / p}
$$

holds only when $p=2$ (see also [6]). However, one can obtain positive results by averaging in time. In particular in [7] it is proved that if $2 \leq p<\frac{2 D}{D-1}$, then spherically symmetric solutions to $(*)$ with initial velocity $\frac{\partial}{\partial t} u(0, x)=0$ satisfy the estimate

$$
\left\{\frac{1}{T} \int_{0}^{T} \int_{R^{D}}|u(t, x)|^{p} d x d t\right\}^{1 / p} \leq c\left\{\int_{R^{D}}|u(0, x)|^{p} d x\right\}^{1 / p} .
$$


At the critical index $p=\frac{2 D}{D-1}$ the above inequality may fail, but the authors stated a restricted weak-type result, that is a weak-type inequality for characteristic functions. Here we shall prove that at the critical index, $p=\frac{2 D}{D-1}$, the radial solutions satisfy the weak-type inequality

$$
\lambda^{\frac{2 D}{D-1}} \iint_{\left\{t \in(0, T), x \in R^{D},|u(t, x)|>\lambda\right\}} d x d t \leq c T \int_{R^{D}}|u(0, x)|^{\frac{2 D}{D-1}} d x .
$$

The weak-type estimate for $p=\frac{2 D}{D-1}$ together with the strong-type estimate for $p=2$, which follows from an integration by part or an application of the Fourier transform, imply by interpolation the result of Müller and Seeger. Again the functions $|x|^{(1-D) / 2}|| x| \pm t|^{(D-1) / 2} \phi(|x| \pm t)$ show that the above results are sharp.

We shall also consider solutions to $(*)$ with non-vanishing initial velocity. In [9] it is proved that when $\frac{2 D}{D+1}<p<\frac{2 D-4}{D-3}$, for solutions with $u(0, x)=0$ one has the maximal inequality

$$
\left\{\int_{R^{D}}\left(\sup _{0<t<+\infty} \frac{|u(t, x)|}{t}\right)^{p} d x\right\}^{1 / p} \leq c\left\{\int_{R^{D}}\left|\frac{\partial}{\partial t} u(0, x)\right|^{p} d x\right\}^{1 / p} .
$$

It also follows from [1] that at the critical index $p=\frac{2 D}{D+1}$ one has a restricted weak-type result. The above estimate holds for all solutions, not necessarily radial, and as a consequence one obtains the almost everywhere convergence as $t \rightarrow 0+$ of $\frac{u(t, x)}{t}$ to $\frac{\partial}{\partial t} u(0, x)$, if the initial velocity is locally in $\mathbb{L}^{p}\left(R^{D}\right), p>\frac{2 D}{D+1}$. Here we shall prove that if $u(0, x)=0$ and if $\frac{\partial}{\partial t} u(0, x)$ is the characteristic function of a spherically symmetric set, then one has the inequality

$$
\begin{aligned}
& \lambda^{\frac{2 D}{D+1}} \int\left\{x \in R^{D}, \sup _{0<t<+\infty} \frac{|u(t, x)|}{t}>\lambda\right\}^{d x} \\
& \leq c\left(\int_{R^{D}}|x|^{\frac{1-D}{2}}\left|\frac{\partial}{\partial t} u(0, x)\right| d x+\left\{\int_{R^{D}}\left|\frac{\partial}{\partial t} u(0, x)\right|^{\frac{2 D}{D+1}} d x\right\}^{\frac{D+1}{2 D}}\right) .
\end{aligned}
$$

This not only implies the restricted weak-type result at $p=\frac{2 D}{D+1}$, but also a weak-type inequality for functions with bounded support disjoint from the origin. Finally, we shall show that for radial solutions to $(*)$ with $u(0, x)=0$, the almost everywhere convergence of $\frac{u(t, x)}{t}$ to $\frac{\partial}{\partial t} u(0, x)$ holds as soon as this initial value is locally integrable. 
We mentioned before that when the space dimension is odd, then both statements and proofs of the results became simpler. This is perhaps related to the Huygens phenomenon. However, since the method of descent does not preserve spherical symmetry, it is not clear how to deduce the even case from the odd one. Indeed to consider only odd dimensions would have reduced the size of the paper by more than a half.

\section{Radial solutions to the wave equation}

In this section we give the explicit solution of the Cauchy problem $(* *)$, where we write $2 \alpha+2$ for the space dimension $D$. Since it is not necessary to assume in what follows that this number is an integer, we work in this more general context.

Theorem 1.1. Let $\alpha \geq-1 / 2$ and $0<t, x<+\infty$. The solution of the Cauchy problem

$$
\left\{\begin{array}{l}
\frac{\partial^{2}}{\partial t^{2}} u(t, x)=\frac{\partial^{2}}{\partial x^{2}} u(t, x)+\frac{2 \alpha+1}{x} \frac{\partial}{\partial x} u(t, x), \\
u(0, x)=\phi(x), \frac{\partial}{\partial t} u(0, x)=\psi(x),
\end{array}\right.
$$

is given by

$$
\begin{aligned}
& u(t, x)=\int_{0}^{+\infty} \mathbb{K}(t, x, y) \psi(y) d y+\int_{0}^{+\infty} \frac{\partial}{\partial t} \mathbb{K}(t, x, y) \phi(y) d y \\
& + \begin{cases}\frac{(x-t)^{\alpha+1 / 2} \phi(x-t)+(x+t)^{\alpha+1 / 2} \phi(x+t)}{2 x^{\alpha+1 / 2}} & \text { if } 0<t \leq x, \\
\frac{-\sin (\pi \alpha)(t-x)^{\alpha+1 / 2} \phi(t-x)+(t+x)^{\alpha+1 / 2} \phi(t+x)}{2 x^{\alpha+1 / 2}} & \text { if } 0<x \leq t .\end{cases}
\end{aligned}
$$

The two kernels $\mathbb{K}(t, x, y)$ and $\frac{\partial}{\partial t} \mathbb{K}(t, x, y)$ are expressed in terms of hypergeometric functions.

i) If $y<x-t$ or if $y>x+t$, then $\mathbb{K}(t, x, y)=\frac{\partial}{\partial t} \mathbb{K}(t, x, y)=0$.

ii) If $|x-t|<y<x+t$, then

$$
\mathbb{K}(t, x, y)=\frac{1}{2} x^{-\alpha-1 / 2} y^{\alpha+1 / 2} \mathbb{F}\left(1 / 2-\alpha, 1 / 2+\alpha ; 1 ; \frac{t^{2}-(x-y)^{2}}{4 x y}\right)
$$

and

$$
\begin{aligned}
& \frac{\partial}{\partial t} \mathbb{K}(t, x, y) \\
& =\frac{(1 / 2-\alpha)(1 / 2+\alpha)}{4} t x^{-\alpha-3 / 2} y^{\alpha-1 / 2} \mathbb{F}\left(3 / 2-\alpha, 3 / 2+\alpha ; 2 ; \frac{t^{2}-(x-y)^{2}}{4 x y}\right) .
\end{aligned}
$$


iii) If $y<t-x$, then

$$
\begin{aligned}
& \mathbb{K}(t, x, y)=\frac{2^{-2 \alpha-1} \sqrt{\pi}}{\Gamma(1 / 2-\alpha) \Gamma(\alpha+1)} x^{-\alpha-1 / 2} y^{\alpha+1 / 2} \\
& \cdot\left(\frac{4 x y}{t^{2}-(x-y)^{2}}\right)^{\alpha+1 / 2} \mathbb{F}\left(\alpha+1 / 2, \alpha+1 / 2 ; 2 \alpha+1 ; \frac{4 x y}{t^{2}-(x-y)^{2}}\right)
\end{aligned}
$$

and

$$
\begin{aligned}
& \frac{\partial}{\partial t} \mathbb{K}(t, x, y)=\frac{2^{-2 \alpha-2} \sqrt{\pi}}{\Gamma(-1 / 2-\alpha) \Gamma(\alpha+1)} t x^{-\alpha-3 / 2} y^{\alpha-1 / 2} \\
& \cdot\left(\frac{4 x y}{t^{2}-(x-y)^{2}}\right)^{\alpha+3 / 2} \mathbb{F}\left(\alpha+3 / 2, \alpha+1 / 2 ; 2 \alpha+1 ; \frac{4 x y}{t^{2}-(x-y)^{2}}\right) .
\end{aligned}
$$

When the space dimension $2 \alpha+2$ is not an odd integer, at $y=t-x$ the kernel $\mathbb{K}(t, x, y)$ has a logarithmic singularity, $\mathbb{K}(t, x, y) \approx \log |t-x-y|$, and under mild assumptions on the function $\psi(y)$ the integral $\int_{0}^{+\infty} \mathbb{K}(t, x, y) \psi(y) d y$ is well defined. On the other hand, $\frac{\partial}{\partial t} \mathbb{K}(t, x, y)$ has at $y=t-x$ a non-integrable singularity, $\frac{\partial}{\partial t} \mathbb{K}(t, x, y) \approx(t-x-y)^{-1}$, and the integral against this kernel has to be defined in the principal value sense,

$$
\int_{0}^{+\infty} \frac{\partial}{\partial t} \mathbb{K}(t, x, y) \phi(y) d y=\lim _{\varepsilon \rightarrow 0+} \int_{\{0<y<+\infty,|t-x-y|>\varepsilon\}} \frac{\partial}{\partial t} \mathbb{K}(t, x, y) \phi(y) d y .
$$

When the space dimension is an odd integer greater than one, $2 \alpha+2=2 N+1$, that is $\alpha=N-1 / 2$, then $1 / \Gamma( \pm 1 / 2-\alpha)=0$, so that the kernels $\mathbb{K}(t, x, y)$ and $\frac{\partial}{\partial t} \mathbb{K}(t, x, y)$ have support in $\{|x-t|<y<x+t\}$. Also, the hypergeometric functions reduce to Legendre polynomials and the solution formula takes a simpler form.

Corollary 1.2. If $2 \alpha+2=2 N+1$ is an odd integer greater than one, then

$$
\begin{aligned}
u(t, x)= & \frac{(x-t)^{N} \phi(x-t)+(x+t)^{N} \phi(x+t)}{2 x^{N}} \\
& -\frac{1}{2} t x^{-N-1} \int_{|x-t|}^{x+t} y^{N-1} \dot{P}_{N-1}\left(\frac{y^{2}+x^{2}-t^{2}}{2 x y}\right) \phi(y) d y \\
& +\frac{1}{2} x^{-N} \int_{|x-t|}^{x+t} y^{N} P_{N-1}\left(\frac{y^{2}+x^{2}-t^{2}}{2 x y}\right) \psi(y) d y .
\end{aligned}
$$


If $2 \alpha+2=1$ then

$$
\begin{aligned}
u(t, x)= & \frac{\phi(x-t)+\phi(x+t)}{2} \\
& + \begin{cases}\frac{1}{2} \int_{x-t}^{x+t} \psi(y) d y & \text { if } 0<t \leq x, \\
\int_{0}^{t-x} \psi(y) d y+\frac{1}{2} \int_{t-x}^{t+x} \psi(y) d y & \text { if } 0<x \leq t .\end{cases}
\end{aligned}
$$

Observe that

$$
\begin{aligned}
-\frac{1}{2} t x^{-N-1} y^{N-1} \dot{\mathbb{P}}_{N-1}\left(\frac{y^{2}+x^{2}-t^{2}}{2 x y}\right) & =x^{1-2 N} \mathbb{A}(t, x, y), \\
\frac{1}{2} x^{-N} y^{N} \mathbb{P}_{N-1}\left(\frac{y^{2}+x^{2}-t^{2}}{2 x y}\right) & =x^{1-2 N} \mathbb{B}(t, x, y),
\end{aligned}
$$

with $\mathbb{A}(t, x, y)$ and $\mathbb{B}(t, x, y)$ polynomials in $(t, x, y)$, odd and of degree $2 N-3$ and $2 N-1$ in the $y$ variable. A repeated integration by parts then gives the following:

Corollary 1.3. If $2 \alpha+2=2 N+1$ is a non-negative odd integer, then

$$
\begin{aligned}
u(t, x)= & x^{1-2 N} \sum_{j=0}^{2 N-2}\left(a_{j}(t, x) \phi^{(-j)}(x-t)+b_{j}(t, x) \phi^{(-j)}(x+t)\right) \\
& +x^{1-2 N} \sum_{j=1}^{2 N}\left(c_{j}(t, x) \psi^{(-j)}(x-t)+d_{j}(t, x) \psi^{(-j)}(x+t)\right),
\end{aligned}
$$

where $a_{j}(t, x), b_{j}(t, x), c_{j}(t, x), d_{j}(t, x)$, are polynomials in $(t, x), \phi^{(0)}(y)=$ $\phi(y), \psi^{(0)}(y)=\psi(y)$, and $\phi^{(-j)}(y)$ and $\psi^{(-j)}(y)$ are primitives of $\phi^{(1-j)}(y)$ and $\psi^{(1-j)}(y)$.

See also [4] for a result related to this last corollary. As we said, a proof of the theorem based on Riemann's method can be found in [3] or [11], moreover in [2] there is a different proof for odd dimensions. Let us now give our proof.

Proof. The approach is straightforward: we write the solution to the wave equation using Fourier integrals and then explicitly compute these integrals.

Lemma 1.4. Let $J_{\alpha}(z)$ be the Bessel function of the first kind and order $\alpha$. Then

$$
\begin{aligned}
u(t, x)= & \int_{0}^{+\infty} \cos (t z)\left(\int_{0}^{+\infty} \frac{J_{\alpha}(z y)}{(z y)^{\alpha}} \phi(y) y^{2 \alpha+1} d y\right) \frac{J_{\alpha}(x z)}{(x z)^{\alpha}} z^{2 \alpha+1} d z+ \\
& +\int_{0}^{+\infty} \frac{\sin (t z)}{z}\left(\int_{0}^{+\infty} \frac{J_{\alpha}(z y)}{(z y)^{\alpha}} \psi(y) y^{2 \alpha+1} d y\right) \frac{J_{\alpha}(x z)}{(x z)^{\alpha}} z^{2 \alpha+1} d z .
\end{aligned}
$$


Proof. For $\alpha \geq-1 / 2$ and suitable test functions on $R_{+}$one has the FourierBessel-Hankel transform and the inversion formula

$$
\widehat{\phi}(z)=\int_{0}^{+\infty} \frac{J_{\alpha}(z y)}{(z y)^{\alpha}} \phi(y) y^{2 \alpha+1} d y, \phi(x)=\int_{0}^{+\infty} \frac{J_{\alpha}(x z)}{(x z)^{\alpha}} \widehat{\phi}(z) z^{2 \alpha+1} d z .
$$

Since the functions $x \longmapsto(x z)^{-\alpha} J_{\alpha}(x z)$ are eigenfunctions of the radial Laplacian,

$$
\left(\frac{\partial^{2}}{\partial x^{2}}+\frac{2 \alpha+1}{x} \frac{\partial}{\partial x}\right)\left(\frac{J_{\alpha}(x z)}{(x z)^{\alpha}}\right)=-z^{2} \frac{J_{\alpha}(x z)}{(x z)^{\alpha}},
$$

by taking the Fourier-Bessel-Hankel transform with respect to the space variable one reduces the partial differential equation to an ordinary differential equation in the time variable,

$$
\left\{\begin{array}{l}
\frac{\partial^{2}}{\partial t^{2}} \widehat{u}(t, z)=-z^{2} \widehat{u}(t, z), \\
\widehat{u}(0, z)=\widehat{\phi}(z), \frac{\partial}{\partial t} \widehat{u}(0, z)=\widehat{\psi}(z),
\end{array}\right.
$$

with the solution

$$
\widehat{u}(t, z)=\widehat{\phi}(z) \cos (t z)+\widehat{\psi}(z) \frac{\sin (t z)}{z} .
$$

The inversion formula then gives the desired result.

Interchanging the order of integration in the previous lemma, we formally obtain

$$
\begin{aligned}
u(t, x)= & \int_{0}^{+\infty}\left(\int_{0}^{+\infty} \cos (t z) \frac{J_{\alpha}(x z)}{(x z)^{\alpha}} \frac{J_{\alpha}(z y)}{(z y)^{\alpha}}(y z)^{2 \alpha+1} d z\right) \phi(y) d y+ \\
& +\int_{0}^{+\infty}\left(\int_{0}^{+\infty} \frac{\sin (t z)}{z} \frac{J_{\alpha}(x z)}{(x z)^{\alpha}} \frac{J_{\alpha}(z y)}{(z y)^{\alpha}}(y z)^{2 \alpha+1} d z\right) \psi(y) d y \\
& =\int_{0}^{+\infty} \mathbb{H}(t, x, y) \phi(y) d y+\int_{0}^{+\infty} \mathbb{K}(t, x, y) \psi(y) d y .
\end{aligned}
$$

Since $J_{\alpha}(z) \approx \sqrt{2 / \pi z} \cos (z-\alpha \pi / 2-\pi / 4)$, the integral that defines $\mathbb{H}(t, x, y)$ is divergent. Indeed we will see that the associated operator is singular and gives rise to the terms $x^{-\alpha-1 / 2}(x \pm t)^{\alpha+1 / 2} \phi(x \pm t)$. However, formally we have

$$
\int_{0}^{+\infty} \mathbb{H}(t, x, y) \phi(y) d y=\frac{\partial}{\partial t}\left(\int_{0}^{+\infty} \mathbb{K}(t, x, y) \phi(y) d y\right),
$$

and it turns out that the kernel $\mathbb{K}(t, x, y)$ is an integrable function which can be computed explicitly in terms of Legendre functions. 
Lemma 1.5. Assuming $t, x, y>0$, we have

$$
\begin{aligned}
& \mathbb{K}(t, x, y)=x^{-\alpha} y^{\alpha+1} \int_{0}^{+\infty} J_{\alpha}(x z) J_{\alpha}(y z) \sin (t z) d z \\
& =\sqrt{\pi / 2} x^{-\alpha} y^{\alpha+1} \int_{0}^{+\infty} J_{\alpha}(x z) J_{\alpha}(y z) J_{1 / 2}(t z)(t z)^{1 / 2} d z \\
& = \begin{cases}0 & \text { if } y<x-t \text { or } y>x+t \\
\frac{1}{2} x^{-\alpha-1 / 2} y^{\alpha+1 / 2} \mathbb{P}_{\alpha-1 / 2}\left(\frac{y^{2}+x^{2}-t^{2}}{2 x y}\right) & \text { if }|x-t|<y<x+t, \\
\frac{\cos (\pi \alpha)}{\pi} x^{-\alpha-1 / 2} y^{\alpha+1 / 2} \mathbb{Q}_{\alpha-1 / 2}\left(\frac{t^{2}-x^{2}-y^{2}}{2 x y}\right) & \text { if } y<t-x,\end{cases}
\end{aligned}
$$

where $\mathbb{P}_{\alpha-1 / 2}(z)$ and $\mathbb{Q}_{\alpha-1 / 2}(z)$ are the Legendre functions of the first and second kind respectively.

Proof. See [12, (13.46)].

Observe that $\mathbb{K}(t, x, y)=0$ if $|y-x|>t$, or if $y+x<t$ and the space dimension $2 \alpha+2$ is an odd integer. This is also a consequence of the Paley-Wiener theorem since $z \longmapsto(w z)^{-\alpha} J_{\alpha}(w z)$ is an even entire function of exponential type $w$.

When $n$ is a non-negative integer, then $\mathbb{P}_{n}(z)$ is the Legendre polynomial and, if $\alpha=N-1 / 2$,

$$
\mathbb{K}(t, x, y)= \begin{cases}\frac{1}{2} x^{-N} y^{N} \mathbb{P}_{N-1}\left(\frac{y^{2}+x^{2}-t^{2}}{2 x y}\right) & \text { if }|x-t|<y<x+t, \\ 0 & \text { otherwise. }\end{cases}
$$

Moreover, since $\mathbb{P}_{n}(1)=1$ and $\mathbb{P}_{n}(-1)=(-1)^{n}$,

$$
\begin{aligned}
& \lim _{y \rightarrow(t-x)+} \mathbb{K}(t, x, y)=-\frac{1}{2} x^{-N}(x-t)^{N}, \\
& \lim _{y \rightarrow(x-t)+} \mathbb{K}(t, x, y)=\frac{1}{2} x^{-N}(x-t)^{N}, \\
& \lim _{y \rightarrow(x+t)-} \mathbb{K}(t, x, y)=\frac{1}{2} x^{-N}(x+t)^{N} .
\end{aligned}
$$

Differentiating $\frac{\partial}{\partial t}\left(\frac{1}{2} x^{-N} \int_{|x-t|}^{x+t} y^{N} \mathbb{P}_{N-1}\left(\frac{y^{2}+x^{2}-t^{2}}{2 x y}\right) \phi(y) d y\right)$ we obtain the formula in the first corollary. The proof of the theorem is a bit more complicated.

If $v$ is not an integer, then $\mathbb{P}_{v}(z)$ is regular in the complex plane cut along $[-\infty,-1]$; one has $\mathbb{P}_{v}(1)=1$, but there is a logarithmic singularity in $z=-1$. 
Indeed it is possible to write the Legendre functions in terms of hypergeometric functions,

$$
\begin{aligned}
& \mathbb{P}_{v}(z)=\mathbb{F}\left(-v, v+1 ; 1 ; \frac{1-z}{2}\right), \\
& \mathbb{Q}_{v}(z)=\frac{\sqrt{\pi} \Gamma(v+1)}{\Gamma(\nu+3 / 2)}(2 z)^{-v-1} \mathbb{F}\left(v / 2+1 / 2, v / 2+1 ; v+3 / 2 ; z^{-2}\right) .
\end{aligned}
$$

See [5, (7.3)]. Hence, if $|x-t|<y<x+t$ we have

$$
\begin{aligned}
& \mathbb{K}(t, x, y)=\frac{1}{2} x^{-\alpha-1 / 2} y^{\alpha+1 / 2} \mathbb{P}_{\alpha-1 / 2}\left(\frac{y^{2}+x^{2}-t^{2}}{2 x y}\right) \\
& =\frac{1}{2} x^{-\alpha-1 / 2} y^{\alpha+1 / 2} \mathbb{F}\left(1 / 2-\alpha, 1 / 2+\alpha ; 1 ; \frac{t^{2}-(x-y)^{2}}{4 x y}\right) .
\end{aligned}
$$

Similarly, by applying a quadratic transformation to the hypergeometric function, see [5, (9.6.17)], if $0<y<t-x$ we obtain

$$
\begin{aligned}
& \mathbb{K}(t, x, y)=\frac{\cos (\alpha \pi)}{\pi} x^{-\alpha-1 / 2} y^{\alpha+1 / 2} \mathbb{Q}_{\alpha-1 / 2}\left(\frac{t^{2}-x^{2}-y^{2}}{2 x y}\right) \\
& =\frac{2^{-2 \alpha-1} \sqrt{\pi}}{\Gamma(1 / 2-\alpha) \Gamma(\alpha+1)} x^{-\alpha-1 / 2} y^{\alpha+1 / 2}\left(\frac{4 x y}{t^{2}-(x-y)^{2}}\right)^{\alpha+1 / 2} \\
& \cdot \mathbb{F}\left(\alpha+1 / 2, \alpha+1 / 2 ; 2 \alpha+1 ; \frac{4 x y}{t^{2}-(x-y)^{2}}\right) .
\end{aligned}
$$

As it will be seen in a moment, the kernel $\mathbb{K}(t, x, y)$ is locally integrable and when differentiated, $\frac{\partial}{\partial t}\left(\int_{0}^{+\infty} \mathbb{K}(t, x, y) \phi(y) d y\right)$ gives $\int_{0}^{+\infty} \frac{\partial}{\partial t} \mathbb{K}(t, x, y) \phi(y) d y$ plus some terms related to the singularities of the kernel at the points $y= \pm x \pm t$. tions,

To evaluate $\frac{\partial}{\partial t} \mathbb{K}(t, x, y)$ it is enough to differentiate the hypergeometric func-

$$
\begin{aligned}
& \frac{\partial}{\partial z} \mathbb{F}(a, b ; c ; z)=\frac{a b}{c} \mathbb{F}(a+1, b+1 ; c+1 ; z), \\
& \frac{\partial}{\partial z}\left(z^{a} \mathbb{F}(a, b ; c ; z)\right)=a z^{a-1} \mathbb{F}(a+1, b ; c ; z) .
\end{aligned}
$$

Finally, we need to understand the behavior of the kernel $\mathbb{K}(t, x, y)$ in a neighborhood of the singular points $y=x+t, y=x-t, y=t-x$. In order to do this, we recall that if $a, b \neq 0,-1,-2, \ldots$ and $z \rightarrow 1-$, then the hypergeometric function has the asymptotic expansion

$\mathbb{F}(a, b ; a+b ; z)=\frac{\Gamma(a+b)}{\Gamma(a) \Gamma(b)}(-\log (1-z)+2 \Psi(1)-\Psi(a)-\Psi(b)+o(1))$,

where $\Gamma(z)$ is the gamma function and $\Psi(z)$ is its logarithmic derivative (see [5, (9.7)]. 
Lemma 1.6. Assume that $2 \alpha+2$ is not an odd integer.

i) Let $0<t<x$. Then, if $y \rightarrow(x-t)+$,

$$
\lim _{y \rightarrow(x-t)+} \mathbb{K}(t, x, y)=\frac{1}{2} x^{-\alpha-1 / 2}(x-t)^{\alpha+1 / 2},
$$

and, if $y \rightarrow(x+t)-$,

$$
\lim _{y \rightarrow(x+t)-} \mathbb{K}(t, x, y)=\frac{1}{2} x^{-\alpha-1 / 2}(x+t)^{\alpha+1 / 2} .
$$

ii) Let $0<x<t$. Then, if $y \rightarrow(t-x)+$,

$\mathbb{K}(t, x, y)$

$$
\begin{aligned}
& =\frac{\cos (\pi \alpha)}{2 \pi} x^{-\alpha-1 / 2}(t-x)^{\alpha+1 / 2}\left(-\log (y-(t-x))+\log \left(\frac{x(t-x)}{t}\right)\right. \\
& +\log 2+2 \Psi(1)-\Psi(1 / 2-\alpha)-\Psi(1 / 2+\alpha)+o(1)),
\end{aligned}
$$

and, if $y \rightarrow(t-x)-$,

$\mathbb{K}(t, x, y)$

$$
\begin{aligned}
& =\frac{\cos (\pi \alpha)}{2 \pi} x^{-\alpha-1 / 2}(t-x)^{\alpha+1 / 2}\left(-\log ((t-x)-y)+\log \left(\frac{x(t-x)}{t}\right)\right. \\
& +\log 2+2 \Psi(1)-2 \Psi(1 / 2+\alpha)+o(1)) .
\end{aligned}
$$

Proof. When $t^{2}=(x-y)^{2}$ one has $\frac{y^{2}+x^{2}-t^{2}}{2 x y}=1$, and since $\left.\mathbb{P}_{v}(1)=1, \mathrm{i}\right)$ follows.

When $t^{2}=(x+y)^{2}$, one has $\frac{y^{2}+x^{2}-t^{2}}{2 x y}=-1$, and $\mathbb{P}_{v}(z)$ has a logarithmic singularity in $z=-1$. Indeed if $y \rightarrow(t-x)+$, then $\frac{t^{2}-(x-y)^{2}}{4 x y} \rightarrow 1-$ and we have

$$
\begin{aligned}
& \mathbb{K}(t, x, y)=\frac{1}{2} x^{-\alpha-1 / 2} y^{\alpha+1 / 2} \mathbb{F}\left(1 / 2-\alpha, 1 / 2+\alpha ; 1 ; \frac{t^{2}-(x-y)^{2}}{4 x y}\right) \\
& =\frac{\cos (\pi \alpha)}{2 \pi} x^{-\alpha-1 / 2} y^{\alpha+1 / 2} \\
& \cdot\left(-\log \left(\frac{(x+y)^{2}-t^{2}}{4 x y}\right)+2 \Psi(1)-\Psi(1 / 2-\alpha)-\Psi(1 / 2+\alpha)+o(1)\right) \\
& =\frac{\cos (\pi \alpha)}{2 \pi} x^{-\alpha-1 / 2}(t-x)^{\alpha+1 / 2}\left(-\log (y-(t-x))+\log \left(\frac{x(t-x)}{t}\right)\right. \\
& +\log 2+2 \Psi(1)-\Psi(1 / 2-\alpha)-\Psi(1 / 2+\alpha)+o(1)) .
\end{aligned}
$$


Similarly, if $y \rightarrow(t-x)-$, then $\frac{4 x y}{t^{2}-(x-y)^{2}} \rightarrow 1-$ and we have $\mathbb{K}(t, x, y)=\frac{2^{-2 \alpha-1} \sqrt{\pi}}{\Gamma(1 / 2-\alpha) \Gamma(\alpha+1)} x^{-\alpha-1 / 2} y^{\alpha+1 / 2}$

$\cdot\left(\frac{4 x y}{t^{2}-(x-y)^{2}}\right)^{\alpha+1 / 2} \mathbb{F}\left(\alpha+1 / 2, \alpha+1 / 2 ; 2 \alpha+1 ; \frac{4 x y}{t^{2}-(x-y)^{2}}\right)$

$=\frac{\cos (\pi \alpha)}{2 \pi} x^{-\alpha-1 / 2} y^{\alpha+1 / 2}\left(\frac{4 x y}{t^{2}-(x-y)^{2}}\right)^{\alpha+1 / 2}$

$\cdot\left(-\log \left(\frac{t^{2}-(x+y)^{2}}{t^{2}-(x-y)^{2}}\right)+2 \Psi(1)-2 \Psi(1 / 2+\alpha)+o(1)\right)$

$=\frac{\cos (\pi \alpha)}{2 \pi} x^{-\alpha-1 / 2}(t-x)^{\alpha+1 / 2}\left(-\log ((t-x)-y)+\log \left(\frac{x(t-x)}{t}\right)\right.$

$+\log 2+2 \Psi(1)-2 \Psi(1 / 2+\alpha)+o(1))$.

In particular, when $y$ passes from $(t-x)-$ to $(t-x)+$, the function

$$
\mathbb{K}(t, x, y)+\frac{\cos (\pi \alpha)}{2 \pi} x^{-\alpha-1 / 2}(t-x)^{\alpha+1 / 2} \log |(t-x)-y|
$$

makes a jump of $\frac{\sin (\pi \alpha)}{2} x^{-\alpha-1 / 2}(t-x)^{\alpha+1 / 2}$, from

$$
\begin{aligned}
& \frac{\cos (\pi \alpha)}{2 \pi} x^{-\alpha-1 / 2}(t-x)^{\alpha+1 / 2} \\
& \cdot\left(\log \left(\frac{x(t-x)}{t}\right)+\log 2+2 \Psi(1)-2 \Psi(1 / 2+\alpha)\right)
\end{aligned}
$$

to

$$
\begin{aligned}
& \frac{\cos (\pi \alpha)}{2 \pi} x^{-\alpha-1 / 2}(t-x)^{\alpha+1 / 2} \\
& \cdot\left(\log \left(\frac{x(t-x)}{t}\right)+\log 2+2 \Psi(1)-\Psi(1 / 2-\alpha)-\Psi(1 / 2+\alpha)\right) .
\end{aligned}
$$

When differentiated with respect to $t$, the term $\log |(t-x)-y|$ gives the singular kernel $(t-x-y)^{-1}$, while the jump produces the term

$$
-\frac{\sin (\pi \alpha)}{2} x^{-\alpha-1 / 2}(t-x)^{\alpha+1 / 2} \phi(t-x) .
$$

Similarly, when differentiated, the jumps at $y=x \pm t$ produce the terms

$$
\frac{1}{2} x^{-\alpha-1 / 2}(x \pm t)^{\alpha+1 / 2} \phi(x \pm t) .
$$

The proof of the theorem is then completed. 
Remark 1. The Legendre functions satisfy the three terms recurrence relations

$$
\begin{aligned}
& (v+1) \mathbb{P}_{v+1}(z)-(2 v+1) z \mathbb{P}_{v}(z)+v \mathbb{P}_{v-1}(z)=0, \\
& v \mathbb{P}_{v+1}(z)-(2 v+1) z \dot{\mathbb{P}}_{v}(z)+(v+1) \dot{\mathbb{P}}_{v-1}(z)=0,
\end{aligned}
$$

and the same for $\mathbb{Q}_{v}(z)$. See [5, (7.8)]. From this it follows that solutions to the wave equation in dimension $2 \alpha+2$ can be expressed in terms of solutions in dimensions $2 \alpha$ and $2 \alpha-2$.

Remark 2. When $\alpha+1 / 2=N$ is an integer, then the kernel $\mathbb{K}(t, x, y)$ vanishes outside $\{|x-t|<y<x+t\}$ and $|\mathbb{K}(t, x, y)| \leq 2^{-1} x^{-N} y^{N}$. If, for every $\delta>\varepsilon>0$, we have $\int_{\varepsilon}^{\delta}|\psi(y)| d y<+\infty$, then the integral $\int_{0}^{+\infty} \mathbb{K}(t, x, y) \psi(y) d y$ is well defined and it gives a continuous function in $\{0<t \neq x<+\infty\}$. Moreover, if $\int_{0}^{\delta}|\psi(y)| y^{N} d y<+\infty$, then $\int_{0}^{+\infty} \mathbb{K}(t, x, y) \psi(y) d y$ is well defined and it gives a continuous function in all $\{0<t, x<+\infty\}$.

When $\alpha+1 / 2$ is not an integer then the situation is a bit more complicated, since $\mathbb{K}(t, x, y)$ is supported in $\{\max \{0, x-t\}<y<x+t\}$ with a logarithmic singularity at $y=t-x$. Anyhow, if $\int_{0}^{\delta}|\psi(y)| y^{\alpha+1 / 2} d y<+\infty$ and, for every $z$, $\int_{\{|z-y|<|z| / 2\}}|\log | z-y|||\psi(y)| d y<+\infty$, then $\int_{0}^{+\infty} \mathbb{K}(t, x, y) \psi(y) d y$ is well defined for every $t$ and $x$.

Remark 3. When $\alpha+1 / 2=N$ is an integer and $\int_{\varepsilon}^{\delta}|\phi(y)| d y<+\infty$ for every $\delta>\varepsilon>0$, then the integral $\int_{0}^{+\infty} \frac{\partial}{\partial t} \mathbb{K}(t, x, y) \phi(y) d y$ is well defined and it gives a continuous function in $\{0<t \neq x<+\infty\}$. If $\int_{0}^{\delta}|\phi(y)| y^{N-1} d y<+\infty$, then $\int_{0}^{+\infty} \frac{\partial}{\partial t} \mathbb{K}(t, x, y) \phi(y) d y$ is well defined and it gives a continuous function in all $\{0<t, x<+\infty\}$.

When $\alpha+1 / 2$ is not an integer, then $\frac{\partial}{\partial t} \mathbb{K}(t, x, y)$ has a singularity $(t-x-y)^{-1}$. If for every $\delta>0$ we have $\int_{0}^{\delta}|\phi(y)| y^{2 \alpha+1} d y<+\infty$, then $\int_{0}^{+\infty} \frac{\partial}{\partial t} \mathbb{K}(t, x, y) \phi(y) d y$ can be defined almost everywhere in a principal value sense.

Remark 4. Assume that $\int_{\varepsilon}^{\delta}|\psi(y)| d y<+\infty$ and $\int_{0}^{\delta}|\phi(y)| y^{2 \alpha+1} d y<+\infty$. By the previous remarks, if $0<x<+\infty$ and $t \rightarrow 0+$ we have

$$
\lim _{t \rightarrow 0+} \int_{0}^{+\infty} \mathbb{K}(t, x, y) \psi(y) d y=0, \lim _{t \rightarrow 0+} \int_{0}^{+\infty} \frac{\partial}{\partial t} \mathbb{K}(t, x, y) \phi(y) d y=0 .
$$


Also, if $\phi(y)$ is continuous at $x$, then

$$
\lim _{t \rightarrow 0+} \frac{(x-t)^{\alpha+1 / 2} \phi(x-t)+(x+t)^{\alpha+1 / 2} \phi(x+t)}{2 x^{\alpha+1 / 2}}=\phi(x) .
$$

Hence $u(t, x) \rightarrow \phi(x)$ when $t \rightarrow 0+$. Similarly, if $\phi(y)$ is differentiable at $x$ and $\psi(y)$ is continuous at $x$, then $\frac{u(t, x)-u(0, x)}{t} \rightarrow \psi(x)$.

\section{Weak-type estimates}

As an application of the above theorem, in this section we prove a weak-type estimate for spherically symmetric solutions to the wave equation with zero initial velocity. See [8] for the definition and some properties of operators of strong-type, weak-type, restricted weak-type, and for the relation between these definitions and Lorentz spaces.

Theorem 2.1. Let $\alpha>-1 / 2$. Then there exists a constant $c$ such that for every $\lambda>0$ and every test function $\phi(x)$, the solution of the Cauchy problem

$$
\left\{\begin{array}{l}
\frac{\partial^{2}}{\partial t^{2}} u(t, x)=\frac{\partial^{2}}{\partial x^{2}} u(t, x)+\frac{2 \alpha+1}{x} \frac{\partial}{\partial x} u(t, x), \\
u(0, x)=\phi(x), \frac{\partial}{\partial t} u(0, x)=0,
\end{array}\right.
$$

satisfies the weak-type inequality

$$
\lambda^{\frac{2 \alpha+1}{4 \alpha+4}} \iint_{\{0<t<T, 0<x<+\infty,|u(t, x)|>\lambda\}} x^{2 \alpha+1} d x d t \leq c T \int_{0}^{+\infty}|\phi(x)|^{\frac{4 \alpha+4}{2 \alpha+1}} x^{2 \alpha+1} d x .
$$

Proof. In order to prove the theorem we first estimate the kernel $\frac{\partial}{\partial t} \mathbb{K}(t, x, y)$ and when $2 \alpha+2=2 N+1$ is an odd integer this is easy. Indeed if $|x-t|<y<$ $x+t$, then $-1<\frac{y^{2}+x^{2}-t^{2}}{2 x y}<1$ and $\left|\dot{\mathbb{P}}_{N-1}\left(\frac{y^{2}+x^{2}-t^{2}}{2 x y}\right)\right| \leq c$. Hence $\left|\frac{\partial}{\partial t} \mathbb{K}(t, x, y)\right| \leq c t x^{-\alpha-3 / 2} y^{\alpha-1 / 2}$. When $\alpha+1 / 2$ is not an integer the estimates are more delicate.

Lemma 2.2. i) Let $0<t<x$ and $x-t<y<x+t$. Then

$$
\left|\frac{\partial}{\partial t} \mathbb{K}(t, x, y)\right| \leq c t x^{-\alpha-3 / 2} y^{\alpha-1 / 2} .
$$

When $\alpha+1 / 2$ is an integer this estimate also holds in the range $|x-t|<y<$ $x+t$. 
ii) Let $0<x<t$ and $0<\frac{t^{2}-(x-y)^{2}}{4 x y}<1-\varepsilon$, with $0<\varepsilon<1$. Then

$$
\left|\frac{\partial}{\partial t} \mathbb{K}(t, x, y)\right| \leq c t x^{-\alpha-3 / 2} y^{\alpha-1 / 2} .
$$

iii) Let $0<x<t$ and $0<\frac{4 x y}{t^{2}-(x-y)^{2}}<1-\varepsilon$. Then

$$
\left|\frac{\partial}{\partial t} \mathbb{K}(t, x, y)\right| \leq c t x^{-\alpha-3 / 2} y^{\alpha-1 / 2}\left(\frac{4 x y}{t^{2}-(x-y)^{2}}\right)^{\alpha+3 / 2} .
$$

iv) Let $0<x<t$ and $1-\varepsilon<\frac{t^{2}-(x-y)^{2}}{4 x y}<1$. Then

$$
\begin{aligned}
& \left|\frac{\partial}{\partial t} \mathbb{K}(t, x, y)+\frac{\cos (\pi \alpha)}{2 \pi} x^{-\alpha-1 / 2}(t-x)^{\alpha+1 / 2}(t-x-y)^{-1}\right| \\
& \leq c t x^{-\alpha-3 / 2} y^{\alpha-1 / 2} \log \left(\frac{4 x y}{(x+y)^{2}-t^{2}}\right) .
\end{aligned}
$$

v) Let $0<x<t$ and $1-\varepsilon<\frac{4 x y}{t^{2}-(x-y)^{2}}<1$. Then

$$
\begin{aligned}
& \left|\frac{\partial}{\partial t} \mathbb{K}(t, x, y)+\frac{\cos (\pi \alpha)}{2 \pi} x^{-\alpha-1 / 2}(t-x)^{\alpha+1 / 2}(t-x-y)^{-1}\right| \\
& \leq c t x^{-\alpha-3 / 2} y^{\alpha-1 / 2} \log \left(\frac{t^{2}-(x-y)^{2}}{t^{2}-(x+y)^{2}}\right) .
\end{aligned}
$$

Proof. We recall that the hypergeometric functions are singular only at one and infinity, in particular these functions are bounded on the intervals $[0,1-\varepsilon]$.

When $0<t<x$ and $x-t<y<x+t$, then $0<\frac{t^{2}-(x-y)^{2}}{4 x y}<1 / 2$. Hence

$$
\begin{aligned}
& \left|\frac{\partial}{\partial t} \mathbb{K}(t, x, y)\right| \\
& =\left|\frac{(1 / 2-\alpha)(1 / 2+\alpha)}{4} t x^{-\alpha-3 / 2} y^{\alpha-1 / 2} \mathbb{F}\left(3 / 2-\alpha, 3 / 2+\alpha ; 2 ; \frac{t^{2}-(x-y)^{2}}{4 x y}\right)\right| \\
& \leq c t x^{-\alpha-3 / 2} y^{\alpha-1 / 2} .
\end{aligned}
$$

This proves i) and the proofs of ii) and iii) are similar.

In order to prove iv) we recall that

$$
\mathbb{F}(a, b ; c ; z)=(1-z)^{c-a-b} \mathbb{F}(c-a, c-b ; c ; z),
$$


and, if $a, b \neq 0,-1,-2, \ldots$ and $z \rightarrow 1-$,

$$
\begin{aligned}
& \mathbb{F}(a, b ; a+b+1 ; z) \\
& =\frac{\Gamma(a+b+1)}{\Gamma(a+1) \Gamma(b+1)}+\frac{\Gamma(a+b+1)}{\Gamma(a) \Gamma(b)}(1-z) \log (1-z)+\mathcal{O}(1-z) .
\end{aligned}
$$

See $[5,(9.5),(9.7)]$. When $0<x<t$ and $t-x<y<t+x$, then if $y \rightarrow(t-x)+$ we have

$$
\begin{aligned}
& \frac{\partial}{\partial t} \mathbb{K}(t, x, y) \\
& =\frac{(1 / 2-\alpha)(1 / 2+\alpha)}{4} t x^{-\alpha-3 / 2} y^{\alpha-1 / 2} \mathbb{F}\left(3 / 2-\alpha, 3 / 2+\alpha ; 2 ; \frac{t^{2}-(x-y)^{2}}{4 x y}\right) \\
& =\frac{(1 / 2-\alpha)(1 / 2+\alpha)}{4} t x^{-\alpha-3 / 2} y^{\alpha-1 / 2}\left(1-\frac{t^{2}-(x-y)^{2}}{4 x y}\right) \\
& \cdot \mathbb{F}\left(1 / 2+\alpha, 1 / 2-\alpha ; 2 ; \frac{t^{2}-(x-y)^{2}}{4 x y}\right) \\
& =\frac{(1 / 2-\alpha)(1 / 2+\alpha)}{4} t x^{-\alpha-3 / 2} y^{\alpha-1 / 2}\left(\frac{4 x y}{(x+y)^{2}-t^{2}}\right) \\
& +\left(\frac{1}{\Gamma(3 / 2-\alpha) \Gamma(3 / 2+\alpha)}+\frac{1}{\Gamma(1 / 2-\alpha) \Gamma(1 / 2+\alpha)}\left(\frac{(x+y)^{2}-t^{2}}{4 x y}\right)\right. \\
& +\mathcal{O}\left(t x^{-\alpha-3 / 2} y^{\alpha-1 / 2}\right) \\
& \left.+\log \left(\frac{(x+y)^{2}-t^{2}}{4 x y}\right)+\mathcal{O}\left(\frac{(x+y)^{2}-t^{2}}{4 x y}\right)\right) \\
& +\frac{(1 / 2-\alpha)(1 / 2+\alpha)}{4 \Gamma(1 / 2-\alpha) \Gamma(1 / 2+\alpha)} t x^{-\alpha-3 / 2} y^{\alpha-1 / 2} \log \left(\frac{(x+y)^{2}-t^{2}}{4 x y}\right) \\
& =
\end{aligned}
$$

In this computation we have substituted $t-x$ to $y$ and a careful checking shows that the error is of the order of $t-x-y$. This proves iv). 
Similarly, when $0<y<t-x$ and $y \rightarrow(t-x)-$ we have

$$
\begin{aligned}
& \frac{\partial}{\partial t} \mathbb{K}(t, x, y) \\
& =\frac{2^{-2 \alpha-2} \sqrt{\pi}}{\Gamma(-1 / 2-\alpha) \Gamma(\alpha+1)} t x^{-\alpha-3 / 2} y^{\alpha-1 / 2}\left(\frac{4 x y}{t^{2}-(x-y)^{2}}\right)^{\alpha+3 / 2} \\
& \cdot \mathbb{F}\left(\alpha+3 / 2, \alpha+1 / 2 ; 2 \alpha+1 ; \frac{4 x y}{t^{2}-(x-y)^{2}}\right) \\
& =\frac{2^{-2 \alpha-2} \sqrt{\pi}}{\Gamma(-1 / 2-\alpha) \Gamma(\alpha+1)} t x^{-\alpha-3 / 2} y^{\alpha-1 / 2}\left(\frac{4 x y}{t^{2}-(x-y)^{2}}\right)^{\alpha+3 / 2} \\
& \cdot\left(1-\frac{4 x y}{t^{2}-(x-y)^{2}}\right)^{-1} \mathbb{F}\left(\alpha+1 / 2, \alpha-1 / 2 ; 2 \alpha+1 ; \frac{4 x y}{t^{2}-(x-y)^{2}}\right) \\
& =-\frac{\cos (\pi \alpha)}{2 \pi} x^{-\alpha-1 / 2}(t-x)^{\alpha+1 / 2}(t-x-y)^{-1} \\
& +\mathcal{O}\left(t x^{-\alpha-3 / 2} y^{\alpha-1 / 2}\left(1+\log \left(\frac{t^{2}-(x-y)^{2}}{t^{2}-(x+y)^{2}}\right)\right)\right) .
\end{aligned}
$$

Again, we have substituted $t-x$ to $y$, with an error of the order of $t-x-y$. This proves $\mathrm{v}$ ).

By the explicit formula of solutions, $u(t, x)$ is sum of terms of the form $x^{-\alpha-1 / 2}(x \pm t)^{\alpha+1 / 2} \phi(x \pm t)$ and an integral term $\int_{0}^{+\infty} \frac{\partial}{\partial t} \mathbb{K}(t, x, y) \phi(y) d y$.

By the previous lemma, when $0<t<x$, then

$$
\left|\int_{0}^{+\infty} \frac{\partial}{\partial t} \mathbb{K}(t, x, y) \phi(y) d y\right| \leq c t x^{-\alpha-3 / 2} \int_{x-t}^{x+t} y^{\alpha-1 / 2}|\phi(y)| d y .
$$

Similarly, when $0<x<t$ and $0<\frac{t^{2}-(x-y)^{2}}{4 x y}<1-\varepsilon$, then

$$
\left|\int_{\left\{0<\frac{t^{2}-(x-y)^{2}}{4 x y}<1-\varepsilon\right\}} \frac{\partial}{\partial t} \mathbb{K}(t, x, y) \phi(y) d y\right| \leq c t x^{-\alpha-3 / 2} \int_{t-x}^{t+x} y^{\alpha-1 / 2}|\phi(y)| d y .
$$

When $0<x<t$ and $0<\frac{4 x y}{t^{2}-(x-y)^{2}}<1-\varepsilon$, then

$$
\begin{aligned}
& \left|\int_{\left\{0<\frac{4 x y}{t^{2}-(x-y)^{2}}<1-\varepsilon\right\}} \frac{\partial}{\partial t} \mathbb{K}(t, x, y) \phi(y) d y\right| \\
& \leq c \int_{\left\{0<\frac{4 x y}{t^{2}-(x-y)^{2}}<1-\varepsilon\right\}} t x^{-\alpha-3 / 2} y^{\alpha-1 / 2}\left(\frac{4 x y}{t^{2}-(x-y)^{2}}\right)^{\alpha+3 / 2}|\phi(y)| d y \\
& \leq c t(t-x)^{-\alpha-3 / 2} \int_{\left\{0<\frac{4 x y}{t^{2}-(x-y)^{2}}<1-\varepsilon\right\}}(t+x-y)^{-\alpha-3 / 2} y^{2 \alpha+1}|\phi(y)| d y .
\end{aligned}
$$


Finally, when $0<x<t$ and $1-\varepsilon<\frac{t^{2}-(x-y)^{2}}{4 x y}<\frac{1}{1-\varepsilon}$, then

$$
\begin{aligned}
& \left|\int_{\left\{1-\varepsilon<\frac{t^{2}-(x-y)^{2}}{4 x y}<\frac{1}{1-\varepsilon}\right\}} \frac{\partial}{\partial t} \mathbb{K}(t, x, y) \phi(y) d y\right| \\
& \leq\left|\frac{\cos (\pi \alpha)}{2 \pi} x^{-\alpha-1 / 2}(t-x)^{\alpha+1 / 2} \int_{\left\{1-\varepsilon<\frac{t^{2}-(x-y)^{2}}{4 x y}<\frac{1}{1-\varepsilon}\right\}} \frac{\phi(y)}{t-x-y} d y\right| \\
& +c t x^{-\alpha-3 / 2} \int_{\left\{1-\varepsilon<\frac{t^{2}-(x-y)^{2}}{4 x y}<1\right\}} y^{\alpha-1 / 2} \log \left(\frac{4 x y}{(x+y)^{2}-t^{2}}\right)|\phi(y)| d y \\
& +c t x^{-\alpha-3 / 2} \int_{\left\{1-\varepsilon<\frac{4 x y}{t^{2}-(x-y)^{2}}<1\right\}} y^{\alpha-1 / 2} \log \left(\frac{t^{2}-(x-y)^{2}}{t^{2}-(x+y)^{2}}\right)|\phi(y)| d y .
\end{aligned}
$$

Define

$$
\begin{aligned}
& \mathbb{A} \phi(t, x)=x^{-\alpha-1 / 2}|x \pm t|^{\alpha+1 / 2}|\phi(x \pm t)|, \\
& \mathbb{B} \phi(t, x)=t x^{-\alpha-3 / 2} \int_{|x-t|}^{x+t} y^{\alpha-1 / 2}|\phi(y)| d y, \\
& \mathbb{C} \phi(t, x)=t(t-x)^{-\alpha-3 / 2} \int_{\left\{0<\frac{4 x y}{t^{2}-(x-y)^{2}}<1-\varepsilon\right\}}(t+x-y)^{-\alpha-3 / 2} y^{2 \alpha+1}|\phi(y)| d y, \\
& \mathbb{D} \phi(t, x)=t x^{-\alpha-3 / 2} \int_{\left\{1-\varepsilon<\frac{4 x y}{t^{2}-(x-y)^{2}}<1\right\}} y^{\alpha-1 / 2} \log \left(\frac{t^{2}-(x-y)^{2}}{t^{2}-(x+y)^{2}}\right)|\phi(y)| d y, \\
& \mathbb{E} \phi(t, x)=t x^{-\alpha-3 / 2} \int_{\left\{1-\varepsilon<\frac{t^{2}-(x-y)^{2}}{4 x y}<1\right\}} y^{\alpha-1 / 2} \log \left(\frac{4 x y}{(x+y)^{2}-t^{2}}\right)|\phi(y)| d y, \\
& \mathbb{F} \phi(t, x)=x^{-\alpha-1 / 2}(t-x)^{\alpha+1 / 2} \int_{\left\{1-\varepsilon<\frac{t^{2}-(x-y)^{2}}{4 x y}<\frac{1}{1-\varepsilon}\right\}} \frac{\phi(y)}{t-x-y} d y .
\end{aligned}
$$

In order to prove the theorem it suffices to prove that these operators satisfy weak-type estimates, but observe that if $2 \alpha+2$ is an odd integer one has to consider only the first two operators. The rescaling $v(t, x)=u(T t, T x)$ shows that we can assume $T=1$ and we can also assume $\varepsilon$ positive but small.

Lemma 2.3. Let

$$
\mathbb{A} \phi(t, x)=x^{-\alpha-1 / 2}|x \pm t|^{\alpha+1 / 2}|\phi(x \pm t)| .
$$

Then

$$
\lambda^{\frac{4 \alpha+4}{2 \alpha+1}} \iint_{\{0<t<1,0<x<+\infty, \mathbb{A} \phi(t, x)>\lambda\}} x^{2 \alpha+1} d x d t \leq c \int_{0}^{+\infty}|\phi(x)|^{\frac{4 \alpha+4}{2 \alpha+1}} x^{2 \alpha+1} d x .
$$


Proof. Split $\phi(x)$ into $\zeta(x)+\theta(x)$, with $\zeta(x)=\phi(x) \cdot \chi_{\{|x| \leq 3\}}(x)$ and $\theta(x)=$ $\phi(x) \cdot \chi_{\{|x|>3\}}(x)$. It suffices to consider the contributions of $\zeta(x)$ and $\theta(x)$ separately.

Since $\theta(x)=0$ when $|x| \leq 3$, if $0<t<1$ we have

$$
x^{-\alpha-1 / 2}|x \pm t|^{\alpha+1 / 2}|\theta(x \pm t)| \leq c|\theta(x \pm t)|,
$$

and, for every $p$,

$$
\begin{aligned}
& \iint_{\{0<t<1,0<x<+\infty, \mathbb{A} \theta(t, x)>\lambda\}} x^{2 \alpha+1} d x d t \\
& \leq \iint_{\{0<t<1,0<x<+\infty,|\theta(x \pm t)|>c \lambda\}} x^{2 \alpha+1} d x d t \\
& \leq c \lambda^{-p} \int_{0}^{+\infty}|\theta(x)|^{p} x^{2 \alpha+1} d x .
\end{aligned}
$$

Now consider the contribution of $\zeta(x)$. Since $\zeta(x)=0$ when $|x|>3$, we have $x^{2 \alpha+2}|\zeta(x)|^{(4 \alpha+4) /(2 \alpha+1)} \leq 3 x^{2 \alpha+1}|\zeta(x)|^{(4 \alpha+4) /(2 \alpha+1)}$ and

$$
\begin{aligned}
& \iint_{\{0<t<1,0<x<+\infty, \mathbb{A} \zeta(t, x)>\lambda\}} x^{2 \alpha+1} d x d t \\
& \leq \int_{0}^{+\infty}\left(\int_{\left\{-\infty<t<+\infty,|x \pm t|^{\alpha+1 / 2}|\zeta(x \pm t)|>\lambda x^{\alpha+1 / 2}\right\}} d t\right) x^{2 \alpha+1} d x \\
& =c \lambda^{-\frac{4 \alpha+4}{2 \alpha+1}} \int_{0}^{+\infty}\left(\int_{\left\{-\infty<y<+\infty,|y|^{\alpha+1 / 2}|\zeta(y)|>z\right\}} d y\right) z^{\frac{2 \alpha+3}{2 \alpha+1}} d z \\
& =c \lambda^{-\frac{4 \alpha+4}{2 \alpha+1}} \int_{-\infty}^{+\infty}\left(|x|^{\alpha+1 / 2}|\zeta(x)|\right)^{\frac{4 \alpha+4}{2 \alpha+1}} d x \\
& \leq c \lambda^{-\frac{4 \alpha+4}{2 \alpha+1}} \int_{0}^{+\infty}|\zeta(x)|^{\frac{4 \alpha+4}{2 \alpha+1}} x^{2 \alpha+1} d x .
\end{aligned}
$$

Lemma 2.4. Let

$$
\mathbb{B} \phi(t, x)=t x^{-\alpha-3 / 2} \int_{|x-t|}^{x+t} y^{\alpha-1 / 2}|\phi(y)| d y .
$$

Then

$$
\lambda^{\frac{4 \alpha+4}{2 \alpha+1}} \iint_{\{0<t<1,0<x<+\infty, \mathbb{B} \phi(t, x)>\lambda\}} x^{2 \alpha+1} d x d t \leq c \int_{0}^{+\infty} \mid \phi(x)^{\frac{4 \alpha+4}{2 \alpha+1}} x^{2 \alpha+1} d x .
$$

Proof. We split the domain of integration into $\{x<t / 2\},\{t / 2 \leq x \leq 2 t\}$, and $\{2 t<x\}$.

If $x>2 t$, then

$$
t x^{-\alpha-3 / 2} \int_{|x-t|}^{x+t} y^{\alpha-1 / 2}|\phi(y)| d y \leq \frac{c}{t} \int_{x-t}^{x+t}|\phi(y)| d y=c \int_{-1}^{1}|\phi(x+t z)| d z .
$$


Hence for every $p$, by Minkowski's inequality,

$$
\begin{aligned}
& \iint_{\{0<t<1,2 t<x<+\infty, \mathbb{B} \phi(t, x)>\lambda\}} x^{2 \alpha+1} d x d t \\
& \leq c \lambda^{-p} \int_{0}^{1} \int_{2 t}^{+\infty}\left(\int_{-1}^{1}|\phi(x+t z)| d z\right)^{p} x^{2 \alpha+1} d x d t \\
& \leq c \lambda^{-p}\left(\int_{-1}^{1}\left\{\int_{0}^{1} \int_{2 t}^{+\infty}|\phi(x+t z)|^{p}(x+t z)^{2 \alpha+1} d x d t\right\}^{1 / p} d z\right)^{p} \\
& \leq c \lambda^{-p} \int_{0}^{+\infty}|\phi(y)|^{p} y^{2 \alpha+1} d y .
\end{aligned}
$$

If $t / 2 \leq x \leq 2 t$, then, by Hölder's inequality,

$$
\begin{aligned}
& t x^{-\alpha-3 / 2} \int_{|x-t|}^{x+t} y^{\alpha-1 / 2}|\phi(y)| d y \\
& \leq c t^{-\alpha-1 / 2} \int_{|x-t|}^{x+t} y^{\alpha-1 / 2}|\phi(y)| d y \\
& \leq c t^{-\alpha-1 / 2}\left\{\int_{|x-t|}^{x+t} \frac{d y}{y}\right\}^{\frac{2 \alpha+3}{4 \alpha+4}}\left\{\int_{|x-t|}^{x+t}|\phi(y)|^{\frac{4 \alpha+4}{2 \alpha+1}} y^{2 \alpha+1} d y\right\}^{\frac{2 \alpha+1}{4 \alpha+4}} \\
& \leq c t^{-\alpha-1 / 2} \log ^{\frac{2 \alpha+3}{4 \alpha+4}}\left(\left|\frac{x+t}{x-t}\right|\right)\left\{\int_{0}^{+\infty}|\phi(y)|^{\frac{4 \alpha+4}{2 \alpha+1}} y^{2 \alpha+1} d y\right\}^{\frac{2 \alpha+1}{4 \alpha+4}} .
\end{aligned}
$$

Hence

$$
\begin{aligned}
& \lambda^{\frac{4 \alpha+4}{2 \alpha+1}} \iint_{\{0<t<1, t / 2<x<2 t, \mathbb{B} \phi(t, x)>\lambda\}} x^{2 \alpha+1} d x d t \\
\leq & \int_{0}^{1} \int_{t / 2}^{2 t}\left(t x^{-\alpha-3 / 2} \int_{|x-t|}^{x+t} y^{\alpha-1 / 2}|\phi(y)| d y\right)^{\frac{4 \alpha+4}{2 \alpha+1}} x^{2 \alpha+1} d x d t \\
\leq & c\left(\int_{0}^{1} \int_{t / 2}^{2 t}\left(t^{-\alpha-1 / 2} \log ^{\frac{2 \alpha+3}{4 \alpha+4}}\left(\left|\frac{x+t}{x-t}\right|\right)\right)^{\frac{4 \alpha+4}{2 \alpha+1}} x^{2 \alpha+1} d x d t\right) \\
& \cdot\left(\int_{0}^{+\infty}|\phi(y)|^{\frac{4 \alpha+4}{2 \alpha+1}} y^{2 \alpha+1} d y\right) \\
\leq & c \int_{0}^{+\infty}|\phi(y)|^{\frac{4 \alpha+4}{2 \alpha+1}} y^{2 \alpha+1} d y .
\end{aligned}
$$

Finally, if $x<t / 2$ and if $\omega>0$, then

$$
\begin{aligned}
& t x^{-\alpha-3 / 2} \int_{|x-t|}^{x+t} y^{\alpha-1 / 2}|\phi(y)| d y \\
& \leq c x^{-\alpha-1 / 2} t^{\alpha+1 / 2-\omega}\left(\frac{1}{2 x} \int_{t-x}^{t+x} y^{\omega}|\phi(y)| d y\right) \\
& \leq x^{-\alpha-1 / 2} \Omega(t),
\end{aligned}
$$


where $\Omega(t)=c t^{\alpha+1 / 2-\omega} M\left[y^{\omega} \phi(y)\right](t)$ and $M$ denotes the Hardy-Littlewood maximal operator which is applied to the function $y^{\omega} \phi(y)$ and evaluated at the point $t$. Now let $\omega$ be such that $-1<(\alpha+1 / 2-\omega)(4 \alpha+4) /(2 \alpha+1)-1<$ $(4 \alpha+4) /(2 \alpha+1)-1$. Then

$$
\begin{aligned}
& \int_{0}^{1} \Omega(t)^{\frac{4 \alpha+4}{2 \alpha+1}} d t \\
& \leq \int_{0}^{+\infty}\left(M\left[y^{\omega} \phi(y)\right](t)\right)^{\frac{4 \alpha+4}{2 \alpha+1}} t^{\frac{(\alpha+1 / 2-\omega)(4 \alpha+4)}{2 \alpha+1}-1} d t \\
& \leq c \int_{0}^{+\infty}\left|y^{\omega} \phi(y)\right|^{\frac{4 \alpha+4}{2 \alpha+1}} y^{\frac{(\alpha+1 / 2-\omega)(4 \alpha+4)}{2 \alpha+1}-1} d y \\
& =c \int_{0}^{+\infty}|\phi(y)|^{\frac{4 \alpha+4}{2 \alpha+1}} y^{2 \alpha+1} d y,
\end{aligned}
$$

and we get

$$
\begin{aligned}
& \iint_{\{0<t<1,0<x<t / 2, \mathbb{B} \phi(t, x)>\lambda\}} x^{2 \alpha+1} d x d t \\
& \leq \iint_{\left\{0<t<1,0<x<+\infty, x^{-\alpha-1 / 2}>\lambda / \Omega(t)\right\}} x^{2 \alpha+1} d x d t \\
& \leq c \lambda^{-(4 \alpha+4) /(2 \alpha+1)} \int_{0}^{1} \Omega(t)^{\frac{4 \alpha+4}{2 \alpha+1}} d t \\
& \leq c \lambda^{-\frac{4 \alpha+4}{2 \alpha+1}} \int_{0}^{+\infty}|\phi(y)|^{\frac{4 \alpha+4}{2 \alpha+1}} y^{2 \alpha+1} d y .
\end{aligned}
$$

As we said, if $2 \alpha+2$ is an odd integer it suffices to estimate the two operators $\mathbb{A}$ and $\mathbb{B}$, and the proof of the theorem stops here. The general case is more involved, since one has to consider also the operators $\mathbb{C}, \mathbb{D}, \mathbb{E}, \mathbb{F}$.

Lemma 2.5. Let

$$
\mathbb{C} \phi(t, x)=t(t-x)^{-\alpha-3 / 2} \int_{\left\{0<\frac{4 x y}{t^{2}-(x-y)^{2}}<1-\varepsilon\right\}}(t+x-y)^{-\alpha-3 / 2} y^{2 \alpha+1}|\phi(y)| d y .
$$

Then

$$
\lambda^{\frac{4 \alpha+4}{2 \alpha+1}} \iint_{\{0<t<1,0<x<t, \mathbb{C} \phi(t, x)>\lambda\}} x^{2 \alpha+1} d x d t \leq c \int_{0}^{+\infty}|\phi(x)|^{\frac{4 \alpha+4}{2 \alpha+1}} x^{2 \alpha+1} d x .
$$

Proof. When $0<x<t \leq 8 x$ and $0<\frac{4 x y}{t^{2}-(x-y)^{2}}<1-\varepsilon$ for some $\varepsilon>0$, then $0<y<(1-\delta)(t-x)$ for some $\delta>0$ and $t(t+x-y)^{-\alpha-3 / 2} \leq c x^{-\alpha-1 / 2}$. 
Hence

$$
\begin{aligned}
& t(t-x)^{-\alpha-3 / 2} \int_{\left\{0<\frac{4 x y}{t^{2}-(x-y)^{2}}<1-\varepsilon\right\}}(t+x-y)^{-\alpha-3 / 2} y^{2 \alpha+1}|\phi(y)| d y \\
& \leq c x^{-\alpha-1 / 2}(t-x)^{\alpha+1 / 2}\left((t-x)^{-2 \alpha-2} \int_{0}^{t-x}|\phi(y)| y^{2 \alpha+1} d y\right) \\
& \leq c x^{-\alpha-1 / 2}(t-x)^{\alpha+1 / 2} \mathbb{M} \phi(t-x),
\end{aligned}
$$

where $\mathbb{M}$ is the Hardy-Littlewood maximal operator on $0<y<+\infty$ with measure $y^{2 \alpha+1} d y$,

$$
\mathbb{M} \phi(z)=\sup _{0 \leq a<z<b}\left\{\left(\int_{a}^{b} y^{2 \alpha+1} d y\right)^{-1}\left(\int_{a}^{b}|\phi(y)| y^{2 \alpha+1} d y\right)\right\} .
$$

Similarly, when $0<8 x \leq t$, we have

$$
\begin{aligned}
& t(t-x)^{-\alpha-3 / 2} \int_{\left\{0<\frac{4 x y}{t^{2}-(x-y)^{2}}<1-\varepsilon\right\}}(t+x-y)^{-\alpha-3 / 2} y^{2 \alpha+1}|\phi(y)| d y \\
& \leq c t(t-x)^{-\alpha-3 / 2}\left(\int_{0}^{t-x}(t+x-y)^{-\alpha-3 / 2} y^{2 \alpha+1} d y\right) \mathbb{M} \phi(t-x) \\
& \leq c x^{-\alpha-1 / 2}(t-x)^{\alpha+1 / 2} \mathbb{M} \phi(t-x) .
\end{aligned}
$$

We have used the fact that the integral of $|\phi(y)|$ against a positive kernel $\kappa(y)$ decreasing around $t-x$ is bounded by the Hardy-Littlewood maximal function $\mathbb{M} \phi(t-x)$ times the integral $\int \kappa(y) y^{2 \alpha+1} d y$.

Hence, with the notation of Lemma 2.3 we have $\mathbb{C} \phi(t, x) \leq c \mathbb{A} \mathbb{M}(x, t)$ and, by that lemma and the boundedness of the Hardy-Littlewood maximal operator,

$$
\begin{aligned}
& \lambda^{\frac{4 \alpha+4}{2 \alpha+1}} \iint_{\{0<t<1,0<x<+\infty, \mathbb{A M} \phi(t, x)>\lambda\}} x^{2 \alpha+1} d x d t \\
& \leq c \int_{0}^{+\infty}|\mathbb{M} \phi(x)|^{\frac{4 \alpha+4}{2 \alpha+1}} x^{2 \alpha+1} d x \\
& \leq c \int_{0}^{+\infty}|\phi(x)|^{\frac{4 \alpha+4}{2 \alpha+1}} x^{2 \alpha+1} d x .
\end{aligned}
$$

Lemma 2.6. Let

$$
\mathbb{D} \phi(t, x)=t x^{-\alpha-3 / 2} \int_{\left\{1-\varepsilon<\frac{4 x y}{t^{2}-(x-y)^{2}}<1\right\}} y^{\alpha-1 / 2} \log \left(\frac{t^{2}-(x-y)^{2}}{t^{2}-(x+y)^{2}}\right)|\phi(y)| d y .
$$

Then

$$
\lambda^{\frac{4 \alpha+4}{2 \alpha+1}} \iint_{\{0<t<1,0<x<t, \mathbb{D} \phi(t, x)>\lambda\}} x^{2 \alpha+1} d x d t \leq c \int_{0}^{+\infty}|\phi(x)|^{\frac{4 \alpha+4}{2 \alpha+1}} x^{2 \alpha+1} d x .
$$


Proof. Assume $0<x<t \leq 8 x$. Then, for some $\delta>0,\left\{1-\varepsilon<\frac{4 x y}{t^{2}-(x-y)^{2}}<1\right\}$ is contained in $\{(1-\delta)(t-x)<y<t-x\}$, and

$$
\begin{aligned}
& t x^{-\alpha-3 / 2} \int_{\left\{1-\varepsilon<\frac{4 x y}{t^{2}-(x-y)^{2}}<1\right\}} y^{\alpha-1 / 2} \log \left(\frac{t^{2}-(x-y)^{2}}{t^{2}-(x+y)^{2}}\right)|\phi(y)| d y \\
& \leq c x^{-\alpha-1 / 2}(t-x)^{\alpha-1 / 2} \int_{(1-\delta)(t-x)}^{t-x} \log \left(\frac{t^{2}-(x-y)^{2}}{t^{2}-(x+y)^{2}}\right)|\phi(y)| d y \\
& \leq c x^{-\alpha-1 / 2}(t-x)^{\alpha-1 / 2}\left(\int_{(1-\delta)(t-x)}^{t-x} \log \left(\frac{t^{2}-(x-y)^{2}}{t^{2}-(x+y)^{2}}\right) d y\right) \mathbb{M} \phi(t-x) \\
& \leq c x^{-\alpha-1 / 2}(t-x)^{\alpha+1 / 2}(1+|\log (t-x)|) \mathbb{M} \phi(t-x),
\end{aligned}
$$

where, as in the previous lemma, $\mathbb{M}$ is the Hardy-Littlewood maximal operator on $0<y<+\infty$ with measure $y^{2 \alpha+1} d y$.

Assume now $0<8 x \leq t$. Then if $\varepsilon$ is small, $\left\{1-\varepsilon<\frac{4 x y}{t^{2}-(x-y)^{2}}<1\right\}$ is contained in $\{t-2 x<y<t-x\}$, and

$$
\begin{aligned}
& t x^{-\alpha-3 / 2} \int_{\left\{1-\varepsilon<\frac{4 x y}{t^{2}-(x-y)^{2}}<1\right\}} y^{\alpha-1 / 2} \log \left(\frac{t^{2}-(x-y)^{2}}{t^{2}-(x+y)^{2}}\right)|\phi(y)| d y \\
& \leq c x^{-\alpha-3 / 2}(t-x)^{\alpha+1 / 2} \int_{t-2 x}^{t-x} \log \left(\frac{t^{2}-(x-y)^{2}}{t^{2}-(x+y)^{2}}\right)|\phi(y)| d y \\
& \leq c x^{-\alpha-3 / 2}(t-x)^{\alpha+1 / 2}\left(\int_{t-2 x}^{t-x} \log \left(\frac{t^{2}-(x-y)^{2}}{t^{2}-(x+y)^{2}}\right) d y\right) \mathbb{M} \phi(t-x) \\
& \leq c x^{-\alpha-1 / 2}(t-x)^{\alpha+1 / 2} \mathbb{M} \phi(t-x) .
\end{aligned}
$$

Hence we have $\mathbb{D} \phi(t, x) \leq c x^{-\alpha-1 / 2}(t-x)^{\alpha+1 / 2}(1+|\log (t-x)|) \mathbb{M} \phi(t-x)$. Let $\Theta(y)=y^{\alpha+1 / 2}(1+|\log (y)|) \mathbb{M} \phi(y)$. We thus have

$$
\begin{aligned}
& \lambda^{\frac{4 \alpha+4}{2 \alpha+1}} \iint_{\{0<t<1,0<x<t, \mathbb{D} \phi(t, x)>\lambda\}} x^{2 \alpha+1} d x d t \\
& \leq \lambda^{\frac{4 \alpha+4}{2 \alpha+1}} \iint_{\left\{0<y<1,0<x<1, c x^{-\alpha-1 / 2} \Theta(y)>\lambda\right\}} x^{2 \alpha+1} d x d y \\
& \leq \lambda^{\frac{4 \alpha+4}{2 \alpha+1}} \int_{0}^{1} \int_{0}^{(c \Theta(y) / \lambda)^{2 /(2 \alpha+1)}} x^{2 \alpha+1} d x d y \\
& \leq c \int_{0}^{1} \Theta(y)^{\frac{4 \alpha+4}{2 \alpha+1}} d y \\
& \leq c \int_{0}^{1}\left(y^{\alpha+1 / 2}(1+|\log (y)|) \mathbb{M} \phi(y)\right)^{\frac{4 \alpha+4}{2 \alpha+1}} d y \\
& \leq c \int_{0}^{1}|\mathbb{M} \phi(y)|^{\frac{4 \alpha+4}{2 \alpha+1}} y^{2 \alpha+1} d y \\
& \leq c \int_{0}^{+\infty}|\phi(x)|^{\frac{4 \alpha+4}{2 \alpha+1}} x^{2 \alpha+1} d x .
\end{aligned}
$$


Lemma 2.7. Let

$$
\mathbb{E} \phi(t, x)=t x^{-\alpha-3 / 2} \int_{\left\{1-\varepsilon<\frac{t^{2}-(x-y)^{2}}{4 x y}<1\right\}} y^{\alpha-1 / 2} \log \left(\frac{4 x y}{(x+y)^{2}-t^{2}}\right)|\phi(y)| d y .
$$

Then

$$
\lambda^{\frac{4 \alpha+4}{2 \alpha+1}} \iint_{\{0<t<1,0<x<t, \mathbb{E} \phi(t, x)>\lambda\}} x^{2 \alpha+1} d x d t \leq c \int_{0}^{+\infty}|\phi(x)|^{\frac{4 \alpha+4}{2 \alpha+1}} x^{2 \alpha+1} d x .
$$

Proof. The proof of this lemma is analogous to the previous one.

Lemma 2.8. Let $\mathbb{S}$ be a sublinear operator on test functions on $0<x<+\infty$ and assume that there exist constants $0<\varepsilon<\delta$ such that the value $\mathbb{S} \phi(x)$ depends only on the values of $\phi(y)$ in the interval $\varepsilon x<y<\delta x$. Also, let $0<p<+\infty$ and $-\infty<\beta, \gamma<+\infty$. Then, if the operator $\mathbb{S}$ is bounded on $\mathbb{L}^{p}\left(R_{+}, x^{\beta} d x\right)$, it is also bounded on $\mathbb{L}^{p}\left(R_{+}, x^{\gamma} d x\right)$.

Proof. Decompose $\phi(x)$ into $\sum_{k=-\infty}^{+\infty} \phi_{k}(x)$, with $\phi_{k}(x)=\phi(x)$ if $2^{k} \leq x<2^{k+1}$ and $\phi_{k}(x)=0$ otherwise. Then $|\mathbb{S} \phi(x)| \leq \sum_{k=-\infty}^{+\infty}\left|\mathbb{S} \phi_{k}(x)\right|$ and by the assumptions on the supports of these functions we have

$$
\begin{aligned}
& \int_{0}^{+\infty}|\mathbb{S} \phi(x)|^{p} x^{\gamma} d x \\
& \leq c \sum_{k=-\infty}^{+\infty} \int_{0}^{+\infty}\left|\mathbb{S} \phi_{k}(x)\right|^{p} x^{\gamma} d x \\
& \leq c \sum_{k=-\infty}^{+\infty} 2^{(\gamma-\beta) k} \int_{0}^{+\infty}\left|\mathbb{S} \phi_{k}(x)\right|^{p} x^{\beta} d x \\
& \leq c \sum_{k=-\infty}^{+\infty} 2^{(\gamma-\beta) k} \int_{0}^{+\infty}\left|\phi_{k}(x)\right|^{p} x^{\beta} d x \\
& \leq c \sum_{k=-\infty}^{+\infty} \int_{0}^{+\infty}\left|\phi_{k}(x)\right|^{p} x^{\gamma} d x=c \int_{0}^{+\infty}|\phi(x)|^{p} x^{\gamma} d x .
\end{aligned}
$$

Lemma 2.9. Let

$$
\mathbb{F} \phi(t, x)=x^{-\alpha-1 / 2}(t-x)^{\alpha+1 / 2} \int_{\left\{1-\varepsilon<\frac{t^{2}-(x-y)^{2}}{4 x y}<\frac{1}{1-\varepsilon}\right\}} \frac{\phi(y)}{t-x-y} d y .
$$

Then

$$
\lambda^{\frac{4 \alpha+4}{2 \alpha+1}} \iint_{\{0<t<1,0<x<t, \mathbb{F} \phi(t, x)>\lambda\}} x^{2 \alpha+1} d x d t \leq c \int_{0}^{+\infty}|\phi(x)|^{\frac{4 \alpha+4}{2 \alpha+1}} x^{2 \alpha+1} d x .
$$


Proof. Let us consider the integral $\int_{\left\{1-\varepsilon<\frac{t^{2}-(x-y)^{2}}{4 x y}<\frac{1}{1-\varepsilon}\right\}} \frac{\phi(y)}{t-x-y} d y$. The interval $\left\{1-\varepsilon<\frac{t^{2}-(x-y)^{2}}{4 x y}<\frac{1}{1-\varepsilon}\right\}$ is not symmetric around $y=t-x$. The integration over the symmetric part is a truncated Hilbert transform, while the integration over the remaining part can be controlled by the Hardy-Littlewood maximal operator.

Given $0<\eta<1$, let

$$
\begin{aligned}
& H_{\eta} \phi(x)=\sup _{0<v<\vartheta<\eta x}\left\{\left|\int_{\{v<|y|<\vartheta\}} \frac{\phi(x-y)}{y} d y\right|\right\}, \\
& M_{\eta} \phi(x)=\sup _{0<\vartheta<\eta x}\left\{\frac{1}{2 \vartheta} \int_{\{|y|<\vartheta\}}|\phi(x-y)| d y\right\},
\end{aligned}
$$

be the truncated maximal Hilbert transform and the truncated maximal HardyLittlewood operator respectively. These operators are bounded on $\mathbb{L}^{p}\left(R_{+}, d x\right)$, $1<p<+\infty$. See [8, (2.3), (6.1)]. Hence, by the above lemma, they are also bounded on $\mathbb{L}^{p}\left(R_{+}, x^{2 \alpha+1} d x\right)$.

Given a small $\varepsilon>0$, there exists $0<\eta<1$ such that

$$
\begin{aligned}
& \left|x^{-\alpha-1 / 2}(t-x)^{\alpha+1 / 2} \int_{\left\{1-\varepsilon<\frac{t^{2}-(x-y)^{2}}{4 x y}<\frac{1}{1-\varepsilon}\right\}} \frac{\phi(y)}{t-x-y} d y\right| \\
& \leq c x^{-\alpha-1 / 2}(t-x)^{\alpha+1 / 2}\left(H_{\eta} \phi(t-x)+M_{\eta} \phi(t-x)\right) \\
& \leq c \mathbb{A}\left(H_{\eta} \phi+M_{\eta} \phi\right)(t, x),
\end{aligned}
$$

where $\mathbb{A}$ is the operator defined in Lemma 2.3. Hence,

$$
\begin{aligned}
& \lambda^{\frac{4 \alpha+4}{2 \alpha+1}} \iint_{\{0<t<1,0<x<t, \mathbb{F} \phi(t, x)>\lambda\}} x^{2 \alpha+1} d x d t \\
& \leq \lambda^{\frac{4 \alpha+4}{2 \alpha+1}} \iint_{\left\{0<t<1,0<x<+\infty, c \mathbb{A}\left(H_{\eta} \phi+M_{\eta} \phi\right)(t, x)>\lambda\right\}} x^{2 \alpha+1} d x d y \\
& \leq c \int_{0}^{+\infty}\left|H_{\eta} \phi(x)+M_{\eta} \phi(x)\right|^{\frac{4 \alpha+4}{2 \alpha+1}} x^{\alpha+1 / 2} d x \\
& \leq c \int_{0}^{+\infty}|\phi(x)|^{\frac{4 \alpha+4}{2 \alpha+1}} x^{2 \alpha+1} d x .
\end{aligned}
$$

The proof of the theorem is then complete.

\section{Restricted weak-type estimates}

In this section we prove a restricted weak-type estimate for spherically symmetric solutions to the wave equation with non-zero initial velocity. 
Theorem 3.1. Let $\alpha \geq-1 / 2$ and let

$$
\left\{\begin{array}{l}
\frac{\partial^{2}}{\partial t^{2}} u(t, x)=\frac{\partial^{2}}{\partial x^{2}} u(t, x)+\frac{2 \alpha+1}{x} \frac{\partial}{\partial x} u(t, x), \\
u(0, x)=0, \frac{\partial}{\partial t} u(0, x)=\psi(x)
\end{array}\right.
$$

Then the maximal function $\sup _{0<t<+\infty} \frac{|u(t, x)|}{t}$ satisfies the weak-type inequality

$$
\begin{aligned}
& \lambda^{\frac{4 \alpha+4}{2 \alpha+3}} \int_{\left\{0<x<+\infty, \sup _{0<t<+\infty} \frac{|u(t, x)|}{t}>\lambda\right\}^{x^{2 \alpha+1}} d x} \\
& \leq c\left(\int_{0}^{+\infty}|\psi(x)| x^{\alpha+1 / 2} d x+\left\{\int_{0}^{+\infty}|\psi(x)|^{\frac{4 \alpha+4}{2 \alpha+3}} x^{2 \alpha+1} d x\right\}^{\frac{2 \alpha+3}{4 \alpha+4}}\right)^{\frac{4 \alpha+4}{2 \alpha+3}} .
\end{aligned}
$$

Proof. Observe that when $\alpha=-1 / 2$ then, by d'Alembert's formula, $\frac{u(t, x)}{t}=$ $\frac{1}{2 t} \int_{x-t}^{x+t} \psi(y) d y$. In this case the maximal operator is controlled by the HardyLittlewood maximal function and the required weak-type inequality follows. In the following we assume $\alpha>-1 / 2$. Since $u(t, x)=\int_{0}^{+\infty} \mathbb{K}(t, x, y) \psi(y) d y$, we start by estimating the kernel $\mathbb{K}(t, x, y)$ and, as before, when $2 \alpha+2=2 N+1$ is an odd integer, the estimates are immediate. Indeed if $|x-t|<y<x+t$, then $\left|\mathbb{P}_{N-1}\left(\frac{y^{2}+x^{2}-t^{2}}{2 x y}\right)\right| \leq c$ and $|\mathbb{K}(t, x, y)| \leq c t x^{-\alpha-1 / 2} y^{\alpha+1 / 2}$. When $\alpha+1 / 2$ is not an integer the estimates are more delicate.

Lemma 3.2. i) Let $0<t<x$ and $x-t<y<x+t$. Then

$$
|\mathbb{K}(t, x, y)| \leq c x^{-\alpha-1 / 2} y^{\alpha+1 / 2} .
$$

When $\alpha+1 / 2$ is an integer this estimate also holds in the range $|x-t|<y<$ $x+t$.

ii) Let $0<x<t$ and $t-x<y<t+x$. Then

$$
|\mathbb{K}(t, x, y)| \leq c x^{-\alpha-1 / 2} y^{\alpha+1 / 2}\left(1+\log \left(\frac{4 x y}{(x+y)^{2}-t^{2}}\right)\right) .
$$

iii) Let $0<x<t$ and $0<y<t-x$. Then

$$
|\mathbb{K}(t, x, y)| \leq c x^{-\alpha-1 / 2} y^{\alpha+1 / 2}\left(\frac{4 x y}{t^{2}-(x-y)^{2}}\right)^{\alpha+1 / 2}\left(1+\log \left(\frac{t^{2}-(x-y)^{2}}{t^{2}-(x+y)^{2}}\right)\right) .
$$


Proof. Since $\mathbb{F}(a, b ; a+b ; z)=-\frac{\Gamma(a+b)}{\Gamma(a) \Gamma(b)} \log (1-z)+\mathcal{O}(1)$, the kernel $\mathbb{K}(t, x, y)$ in a neighborhood of $y=t-x$ has a logarithmic singularity and the lemma follows.

We split the maximal function into $\sup _{0<t \leq x / 2} \frac{|u(t, x)|}{t}$ and $\sup _{x / 2 \leq t<+\infty} \frac{|u(t, x)|}{t}$. It turns out that the first piece is controlled by a truncated Hardy-Littlewood maximal operator, while the second is dominated by a multiple of $x^{-\alpha-3 / 2}$.

\section{Lemma 3.3.}

$$
\sup _{0<t \leq x / 2} t^{-1} \int_{0}^{+\infty}|\mathbb{K}(t, x, y)||\psi(y)| d y \leq c \sup _{0<t \leq x / 2} \frac{1}{2 t} \int_{x-t}^{x+t}|\psi(y)| d y
$$

and

$$
\lambda^{\frac{4 \alpha+4}{2 \alpha+3}} \int_{\left\{0<x<+\infty, \sup _{0<t \leq x / 2} \frac{|u(t, x)|}{t}>\lambda\right\}} x^{2 \alpha+1} d x \leq c \int_{0}^{+\infty}|\psi(x)|^{\frac{4 \alpha+4}{2 \alpha+3}} x^{2 \alpha+1} d x .
$$

Proof. If $0<t \leq x / 2$ and $x-t<y<x+t$, then $|\mathbb{K}(t, x, y)| \leq c$ and

$$
t^{-1} \int_{0}^{+\infty}|\mathbb{K}(t, x, y)||\psi(y)| d y \leq \frac{c}{2 t} \int_{x-t}^{x+t}|\psi(y)| d y .
$$

Since the Hardy-Littlewood maximal operator is bounded on $\mathbb{L}^{p}\left(R_{+}, d x\right)$ if $1<p<+\infty$, by Lemma 2.8 the truncated Hardy-Littlewood maximal operator is bounded on $\mathbb{L}^{p}\left(R_{+}, x^{2 \alpha+1} d x\right)$ and the lemma follows.

\section{Lemma 3.4.}

$$
\begin{aligned}
& \sup _{x / 2 \leq t<+\infty} t^{-1} \int_{0}^{+\infty}|\mathbb{K}(t, x, y)||\psi(y)| d y \\
& \leq c x^{-\alpha-3 / 2}\left(\int_{0}^{+\infty}|\psi(y)| y^{\alpha+1 / 2} d y+\left\{\int_{0}^{+\infty}|\psi(y)|^{\frac{4 \alpha+4}{2 \alpha+3}} y^{2 \alpha+1} d y\right\}^{\frac{2 \alpha+3}{4 \alpha+4}}\right)
\end{aligned}
$$

and

$$
\begin{aligned}
& \lambda^{\frac{4 \alpha+4}{2 \alpha+3}} \int_{\left\{0<x<+\infty, \sup _{x / 2 \leq t<+\infty} \frac{|u(t, x)|}{t}>\lambda\right\}^{x^{2 \alpha+1}} d x} \\
& \leq c\left(\int_{0}^{+\infty}|\psi(x)| x^{\alpha+1 / 2} d x+\left\{\int_{0}^{+\infty}|\psi(x)|^{\frac{4 \alpha+4}{2 \alpha+3}} x^{2 \alpha+1} d x\right\}^{\frac{2 \alpha+3}{4 \alpha+4}}\right)^{\frac{4 \alpha+4}{2 \alpha+3}} .
\end{aligned}
$$

Proof. If $x / 2 \leq t \leq x$, then $|\mathbb{K}(t, x, y)| \leq c x^{-\alpha-1 / 2} y^{\alpha+1 / 2}$ and

$$
t^{-1} \int_{0}^{+\infty}|\mathbb{K}(t, x, y)||\psi(y)| d y \leq c x^{-\alpha-3 / 2} \int_{0}^{+\infty} y^{\alpha+1 / 2}|\psi(y)| d y .
$$


If $x<t$ and if $0<\varepsilon<1$, we split the domain of integration $0<y<t+x$ into four pieces using the increasing function $y \longmapsto \frac{4 x y}{t^{2}-(x-y)^{2}}$. Then $t^{-1} \int_{\left\{0<\frac{4 x y}{t^{2}-(x-y)^{2}}<1-\varepsilon\right\}}|\mathbb{K}(t, x, y)||\psi(y)| d y \leq c x^{-\alpha-3 / 2} \int_{0}^{+\infty} y^{\alpha+1 / 2}|\psi(y)| d y$,
$t^{-1} \int_{\left\{1+\varepsilon<\frac{4 x y}{t^{2}-(x-y)^{2}}<+\infty\right\}}|\mathbb{K}(t, x, y)||\psi(y)| d y \leq c x^{-\alpha-3 / 2} \int_{0}^{+\infty} y^{\alpha+1 / 2}|\psi(y)| d y$.

If $x<t$ and $\varepsilon$ is small, then the set $\left\{1-\varepsilon<\frac{4 x y}{t^{2}-(x-y)^{2}}<1\right\}$ is contained in $\left\{\frac{t-x}{2}<y<t-x\right\}$ and

$$
\begin{aligned}
& t^{-1} \int_{\left\{1-\varepsilon<\frac{4 x y}{t^{2}-(x-y)^{2}}<1\right\}}|\mathbb{K}(t, x, y)||\psi(y)| d y \\
& \leq c t^{-1} \int_{\left\{1-\varepsilon<\frac{4 x y}{t^{2}-(x-y)^{2}}<1\right\}} x^{-\alpha-1 / 2} y^{\alpha+1 / 2} \log \left(\frac{t^{2}-(x-y)^{2}}{t^{2}-(x+y)^{2}}\right)|\psi(y)| d y \\
& \leq c x^{-\alpha-3 / 2} \int_{\frac{t-x}{2}}^{t-x} y^{-\alpha-1 / 2} \log \left(\frac{t^{2}-(x-y)^{2}}{t^{2}-(x+y)^{2}}\right)|\psi(y)| y^{2 \alpha+1} d y \\
& \leq c x^{-\alpha-3 / 2}\left\{\int_{\frac{t-x}{2}}^{t-x} \log ^{\frac{4 \alpha+4}{2 \alpha+1}}\left(\frac{t^{2}-(x-y)^{2}}{t^{2}-(x+y)^{2}}\right) \frac{d y}{y}\right\}^{\frac{2 \alpha+1}{4 \alpha+4}} \\
& \quad \cdot\left\{\int_{0}^{+\infty}|\psi(y)|^{\frac{4 \alpha+4}{2 \alpha+3}} y^{2 \alpha+1} d y\right\}^{\frac{2 \alpha+3}{4 \alpha+4}} \\
& \leq c x^{-\alpha-3 / 2}\left\{\int_{0}^{+\infty}|\psi(y)|^{\frac{4 \alpha+4}{2 \alpha+3}} y^{2 \alpha+1} d y\right\}^{\frac{2 \alpha+3}{4 \alpha+4}} .
\end{aligned}
$$

Similarly, if $x<t$ and $\varepsilon$ is small, then $\left\{1<\frac{4 x y}{t^{2}-(x-y)^{2}}<1+\varepsilon\right\}$ is contained in $\{t-x<y<\min \{t, 2(t-x)\}\}$ and

$$
\begin{aligned}
& t^{-1} \int_{\left\{1<\frac{4 x y}{t^{2}-(x-y)^{2}}<1+\varepsilon\right\}}|\mathbb{K}(t, x, y)||\psi(y)| d y \\
& \leq c x^{-\alpha-3 / 2} \int_{t-x}^{\min \{t, 2(t-x)\}} y^{-\alpha-1 / 2} \log \left(\frac{4 x y}{(x+y)^{2}-t^{2}}\right)|\psi(y)| y^{2 \alpha+1} d y \\
& \leq c x^{-\alpha-3 / 2}\left\{\int_{0}^{+\infty}|\psi(y)|^{\frac{4 \alpha+4}{2 \alpha+3}} y^{2 \alpha+1} d y\right\}^{\frac{2 \alpha+3}{4 \alpha+4}} .
\end{aligned}
$$


Since

$$
\lambda^{\frac{4 \alpha+4}{2 \alpha+3}} \int_{\left\{0<x<+\infty, z x^{-\alpha-3 / 2}>\lambda\right\}} x^{2 \alpha+1} d x=\lambda^{\frac{4 \alpha+4}{2 \alpha+3}} \int_{0}^{(z / \lambda)^{2 /(2 \alpha+3)}} x^{2 \alpha+1} d x=c z^{\frac{4 \alpha+4}{2 \alpha+3}},
$$

the lemma follows.

The proof of the theorem is then complete.

Corollary 3.5. Let

$$
\mathbb{W} \psi(x)=\sup _{0<t<+\infty} \frac{|u(t, x)|}{t}
$$

be the maximal operator defined in the previous theorem. If $\chi_{\varepsilon}(x)$ is the characteristic function of a measurable set $\&$ in $(0,+\infty)$, then

$$
\lambda^{\frac{4 \alpha+4}{2 \alpha+3}} \int_{\{0<x<+\infty, \mathbb{W} \chi \mathcal{E}(x)>\lambda\}} x^{2 \alpha+1} d x \leq c \int_{\mathcal{E}} x^{2 \alpha+1} d x .
$$

In particular, this operator maps the Lorentz space $\mathbb{L}^{\frac{4 \alpha+4}{2 \alpha+3}, 1}\left(R_{+}, x^{2 \alpha+1} d x\right)$ into the Marcinkiewicz space $\mathbb{L}^{\frac{4 \alpha+4}{2 \alpha+3}, \infty}\left(R_{+}, x^{2 \alpha+1} d x\right)$ boundedly.

Proof. By the theorem,

$$
\lambda^{\frac{4 \alpha+4}{2 \alpha+3}} \int_{\left\{0<x<+\infty, \mathbb{W}_{\chi \mathcal{E}}(x)>\lambda\right\}} x^{2 \alpha+1} d x \leq c\left(\int_{\mathcal{E}} x^{\alpha+1 / 2} d x+\left\{\int_{\mathcal{E}} x^{2 \alpha+1} d x\right\}^{\frac{2 \alpha+3}{4 \alpha+4}}\right)^{\frac{4 \alpha+4}{2 \alpha+3}}
$$

but it is easy to see that $\int_{\mathcal{E}} x^{\alpha+1 / 2} d x \leq c\left\{\int_{\mathcal{E}} x^{2 \alpha+1} d x\right\}^{\frac{2 \alpha+3}{4 \alpha+4}}$.

Indeed, let $D>0,1<p, q<+\infty, 1 / p+1 / q=1$. Given a set $\mathcal{E}$ in $R_{+}$, define the set $U$ in $R_{+}$via the equality $\chi_{u}\left(x^{D}\right)=\chi_{\varepsilon}(x)$, so that $|u|=\int_{u} d t=$ $D \int_{\mathcal{E}} x^{D-1} d x$. Then

$$
D \int_{\mathcal{E}} x^{D / p} \frac{d x}{x}=\int_{U} t^{1 / p} \frac{d t}{t} \leq \int_{0}^{|u|} t^{-1 / q} d t=p|\mathcal{U}|^{1 / p}=p\left\{D \int_{\mathcal{E}} x^{D-1} d x\right\}^{1 / p} .
$$

It follows from the above corollary that if $u(0, x)=0$ and if $\frac{\partial}{\partial t} u(0, x)$ is locally in $\mathbb{L}^{p}\left(R_{+}, x^{2 \alpha+1} d x\right), \frac{4 \alpha+4}{2 \alpha+3}<p \leq+\infty$, then for almost every $x$ in $R_{+}$one has $\lim _{t \rightarrow 0+} \frac{u(t, x)-u(0, x)}{t}=\frac{\partial}{\partial t} u(0, x)$. However, this almost everywhere convergence holds for every $1 \leq p \leq+\infty$. 
Corollary 3.6. If the function $\psi(x)$ is integrable in any interval $a<x<b$, $\int_{a}^{b}|\psi(x)| d x<+\infty$, then, for almost every $x$ in this interval one has

$$
\lim _{t \rightarrow 0+} \frac{u(t, x)}{t}=\psi(x)
$$

Proof. In order to prove the almost everywhere convergence of $\frac{u(t, x)}{t}$ as $t \rightarrow$ $0+$, it suffices to obtain the boundedness of the truncated maximal operator $\sup _{0<t<\varepsilon(x)}\left|\frac{u(t, x)}{t}\right|$, where $\varepsilon(x)$ is some positive function. Now recall that waves propagate with finite speed and that $u(t, x)$ depends only on values of $\psi(y)$ in the interval $x-t<y<x+t$. Moreover, we have seen in Lemma 3.3 that when $0<t<x / 2$, then $|\mathbb{K}(t, x, y)| \leq c$, so that if $\varepsilon(x)<x / 2$ we have

$$
\sup _{0<t<\varepsilon(x)}\left|\frac{u(t, x)}{t}\right| \leq c \sup _{0<t<\varepsilon(x)} \frac{1}{2 t} \int_{x-t}^{x+t}|\psi(x)| d y .
$$

Since this truncated Hardy-Littlewood maximal operator is of weak-type $(1,1)$, the corollary follows.

\section{References}

1. Bourgain, J.: Estimations de certaines fonctions maximales. Comptes Rendues Académie des Sciences, Paris 301, 499-502 (1985)

2. Cominardi, A.: Una formula esplicita per soluzioni radiali dell'equazione delle onde. Tesi di Laurea, Università degli Studi di Milano 1996

3. Courant, R., Hilbert, D.: Methods of mathematical physics. Wiley Interscience Publishers 1962

4. Kingston, J.G.: Spherical waves in odd-dimensional space. Quarterly of Applied Mathematics 46, 775-778 (1988)

5. Lebedev, N.: Special functions and their applications. Dover Publications 1972

6. Littman, W.: The wave operator and $\mathrm{L}^{p}$ norms. Journal of Mathematics and Mechanics 12, 55-68 (1963)

7. Müller, D., Seeger, A.: Inequalities for spherically symmetric solutions of the wave equation. Mathematische Zeitschrift 218, 417-426 (1995)

8. Stein, E.M., Weiss, G.: Introduction to Fourier analysis on Euclidean spaces. Princeton University Press 1971

9. Stein, E.M.: Maximal functions: Spherical means. Proceedings National Academy of Sciences U.S.A. 73, 2174-2175 (1976)

10. Tedone, O.: Sull'integrazione dell'equazione $\frac{\partial^{2} \varphi}{\partial t^{2}}-\sum_{1}^{m} \frac{\partial^{2} \varphi}{\partial x_{i}^{2}}=0$. Annali di Matematica
1, 1-23 (1898)

11. Tricomi, F.G.: Equazioni a derivate parziali. Edizioni Cremonese 1957

12. Watson, G.: A treatise on the theory of Bessel functions. Cambridge: Cambridge University Press 1944 